\title{
Correspondence
}

\section{Opportunistic Relay Selection for Cooperative Relaying in Cochannel Interference Contaminated Networks}

\author{
Bo Zhang, Mohammed El-Hajjar, and Lajos Hanzo
}

\begin{abstract}
A multiple-relay assisted cooperative automatic-repeatrequest (CARQ) system is proposed in interference-limited scenario, where multiple-user detection (MUD) receivers using successive interference cancellation (SIC) are employed at the relay nodes (RNs) and the destination node (DN) to combat cochannel interference (CCI). We analyze the outage performance and propose a novel best effort-detection-based opportunistic relay selection (BED-ORS) scheme, which achieves significantly better outage performance than the traditional highest SNR-based ORS scheme offered in the literature. Moreover, considering the effects of the outdated channel state information (CSI), an improved-BED-ORS (I-BED-ORS) scheme is proposed to enhance the robustness against CSI imperfections.
\end{abstract}

Index Terms-Cooperative diversity, multiple access interference, opportunistic relay selection (ORS), outdated channel state information (CSI).

\section{INTRODUCTION}

Cooperative communication is capable of providing spatial diversity for single-antenna aided wireless devices [1], where one or more nodes act as relays for other communicating nodes. Hence, relay selection [2] is an important factor that affects the performance of a cooperative system. As the coordination and synchronization of multiple simultaneously transmitting relay nodes (RNs) constitutes a challenge, the opportunistic relay selection (ORS) [3] may be preferred, which achieves the maximum attainable diversity at lower implementation complexity. From multiple candidate RNs, the ORS may select a single RN that facilitates the highest instantaneous SNR at the destination node (DN) to retransmit the signal from the source node (SN) [3]. In this way, a diversity order equal to the number of RNs $K$ can be achieved [3]. ${ }^{1}$ Recently, several improved ORS algorithms for different scenarios have been proposed. The authors of [4] studied the relay selection for a finite-buffer decode-and-forward cooperative wireless network, and it is shown to achieve better outage performance than the systems without aid of buffers. In [5], the authors investigated and compared the optimal highest SNR-based selection, the geometrybased selection, and the random selection for uniformly distributed

Manuscript received March 24, 2013; revised September 19, 2013 and November 8, 2013; accepted November 11, 2013. This work was supported in part by the Research Councils U.K. under India-U.K. Advanced Technology Centre (IU-ATC), by the European Union under the Concerto project, by the European Research Council under its Advanced Fellow Scheme, and by China-U.K. Scholarship Council. The review of this paper was coordinated by Prof. J.-Y. Chouinard.

B. Zhang is with the School of Electronics and Computer Science, University of Southampton, Southampton SO17 1BJ, U.K., and also with the School of Electronics and Electrical Engineering, National University of Defense Technology, Changsha 410073, China (e-mail: bz2g10@ecs.soton.ac.uk).

M. El-Hajjar and L. Hanzo are with the School of Electronics and Computer Science, University of Southampton, Southampton SO17 1BJ, U.K. (e-mail: meh@ecs.soton.ac.uk; lh@ecs.soton.ac.uk).

Color versions of one or more of the figures in this paper are available online at http://ieeexplore.ieee.org.

Digital Object Identifier 10.1109/TVT.2013.2291162

${ }^{1}$ If the direct link between the $\mathrm{SN}$ and the DN is considered, a maximum diversity order of $(K+1)$ may be achieved. sensor networks, where they have shown that for all proposed schemes, the SNR variance at the DN converges to zero as the number of RNs increases, which effectively mitigate the effects of fading. In [6], an ORS was designed to select multiple relays rather than a single relay to achieve higher end-to-end throughput.

However, the practical networks may suffer from the cochannel interference (CCI), which was not considered in the previous works. Recently, a dual-hop cooperative relaying scheme adopting singleuser detection (SUD) receivers was investigated to mitigate CCI [7][9]. When SUD receivers are adopted at the DN, the authors of [8] show that the traditional ORS based on the highest SNR criterion is outage optimal in a dual-hop relaying system adopting the SUD receivers. However, the SUD receivers treat CCI as noise and suppress it, where the signal structure of the interference is not exploited. In contrast to SUD, multiuser detection relying on successive interference cancellation (MUD-SIC) [10] may achieve better outage performance while introducing moderate implementation complexity [10]. In [11], MUD-SIC receivers were introduced into a cooperative network with multiple SNs, RNs, and DNs, where the transmission rates were optimized for a scenario where multiple RNs access the channel simultaneously.

The conventional MUD-SIC itself is generally adopted at the detection and decoding stage as a physical layer operation to improve the outage performance of the multiuser uplink scheme or a pointto-point (P2P) scheme in the presence of interference, but it has not been intrinsically amalgamated with any ORS design or cooperative automatic-repeat-request (CARQ) scheme as a medium access control (MAC) layer protocol in the existing literature. Therefore, the first research question we consider can be stated as: How to design outageoptimal ORS using MUD-SIC receivers? Furthermore, the outage performance of ORS may degrade due to having a realistic delayed feedback, where the selection decisions are based on the outdated channel state information $(\mathrm{CSI})^{2}$ [12], [13]. However, previous research considers ORS schemes either using outdated CSI assuming interference-free scenarios [12]-[14] or assuming perfect CSI in the presence of CCI [8], [15]. Therefore, our second research question is stated as: How to mitigate the outage performance degradation under outdated CSI for the ORS scheme using MUD-SIC receivers?

Against this background, in this paper, we consider two CARQ systems employing MUD-SIC receivers at the DN. In the first system, which we refer to as $\boldsymbol{S U D}$-aided $\boldsymbol{C A R Q}$, we employ SUD receivers at the RNs. By contrast, in the second system, which we refer to as MUD-SIC-aided CARQ, we employ MUD-SIC receivers at both the DN and the RNs. ${ }^{3}$ Then, we try to address the aforementioned two open problems in the proposed system models, and the novel contributions in this paper may be summarized as follows.

- We analyze the outage performance of the proposed systems and define the criteria for an outage-optimal ORS scheme for systems adopting MUD-SIC receivers at the DN. We design a

\footnotetext{
${ }^{2}$ In this paper, the CSI is defined as the instantaneous channel coefficient capturing the effects of the path loss and fading.

${ }^{3}$ Although a single SN-DN pair is considered in our system, the ORS scheme proposed may be generalized to practical multiuser networks composed of multiple SNs, e.g., in a cellular network, where multiple SN-DN pairs in a cell may be assigned orthogonal channels so that the ORS may be implemented separately for each SN-DN pair, whereas the CCI is dominated by intercell interference.
} 


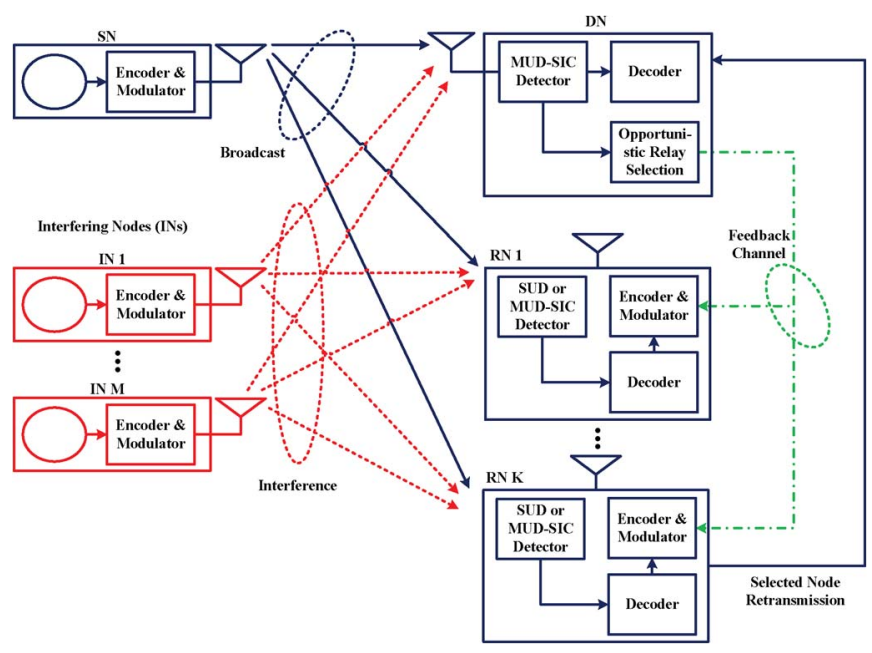

Fig. 1. Schematic of the proposed SUD-aided and MUD-SIC-aided schemes. RNs in the SUD-aided scheme employ the SUD receivers, whereas RNs in the MUD-SIC-aided scheme employ the MUD-SIC receivers.

novel outage-optimal best effort-detection-based-ORS (BEDORS) scheme, which integrates the SIC algorithm into ORS. We demonstrate that the proposed BED-ORS is capable of achieving a better outage performance than the traditional ORS scheme originally designed for SUD receivers [8].

- To enhance the robustness of ORS considering the outdated CSI due to delayed feedback, we design an improved BEDORS (I-BED-ORS), which relies on the statistical information concerning the SN-DN and RN-DN channels to maximize the probability of avoiding an outage. We demonstrate that the I-BED-ORS is more robust against outdated CSI than BED-ORS.

The rest of this paper is organized as follows. The system model is introduced in Section II. In Section III, the outage analysis is detailed, followed by the proposed BED-ORS scheme, as well as our complexity analysis. In Section IV, our I-BED-ORS scheme relying on the outdated CSI is disclosed, whereas our simulation results are provided in Section V, followed by our conclusions in Section VI.

\section{System MODEL}

We consider a network composed of an SN, $K \mathrm{RNs}$, and a DN, as shown in Fig. 1, where $M$ interfering nodes (INs) may access the channel concurrently and introduce CCI. Each node is equipped with a single antenna and operates in a half-duplex mode. We assume a Rayleigh block fading-channel model, where the fading coefficient remains constant for the duration of a packet, and then it is faded independently from one packet to another both in time and space. The noise at the receivers is assumed to be independent zero-mean circularly symmetric complex Gaussian random variables.

At the RNs and the DN, the receiver has to detect the signals transmitted from the desired node, which may be sent by the $\mathrm{SN}$ in the broadcast phase and by the RNs selected for the retransmission phase. The SUD and MUD-SIC receivers are assumed to have perfect CSI for detection and decoding. In the MUD-SIC receiver, both the desired signal and the interfering signals are extracted from the composite received signal. In each iteration, the SIC decoder may remove the interference imposed by the successfully decoded signals from the current composite received signal. This process relies on re-encoding the decoded bit sequence and then remodulating it with the appropriate amplitude and phase adjustment relying on perfect CSI and subtracting it from the interference-infected composite signal. The signal that cannot be error-freely decoded is not subtracted from the composite received signal. It is assumed that error propagation due to erroneous decoding can be avoided with the aid of the cyclic redundancy check (CRC). First, the SN broadcasts its message to all RNs and to the DN. A CRC check is used to test whether the decoding succeeds or fails at the DN. If the decoding is successful, an acknowledgment (ACK) flag is broadcasted by the DN and the SN would initiate the next packet's transmission after receiving the ACK signal. Otherwise, the DN broadcasts the negative-acknowledgment (NACK) flag.

All RNs that overhear the NACK signal from the DN attempt to decode the signal buffered during the SN's broadcast phase. If an RN successfully decodes, it is capable of regenerating the original message and therefore joins the decoding set $D_{s}$. Furthermore, each node in $D_{s}$ sends an ACK signal to the DN to indicate successful decoding and providing the DN with the opportunity to evaluate the CSI [3], based on which the DN implements ORS and selects a retransmission node. Then, the DN notifies the selected node with the aid of a feedback channel, where the node selected re-encodes the message and transmits it. Throughout this paper, it is assumed that the feedback channel and the ACK/NACK flags are error free.

\section{ORS DESIGN}

1) Outage Analysis: An outage is defined as the scenario where the received signal-to-interference-noise power ratio (SINR) experienced at the DN is lower than the predefined threshold $\gamma_{\text {th }}$ that has to be exceeded for successful decoding. When no combining techniques are invoked at the receiver and the erroneous packets are discarded, ${ }^{4}$ the outage probability $P_{l}$ is equal to the probability that both the direct transmission and the retransmission are in outage. Hence, we have $P_{l}=P_{e}^{1} P_{e}^{2}$, where $P_{e}^{1}$ denotes the packet error ratio (PER) of the direct transmission, whereas $P_{e}^{2}$ denotes the PER of the retransmission. As the classic P2P transmission is a basic building block of CARQ, we first present the P2P PER analysis and then we deduce the outage probability of CARQ based on the P2P PER analysis.

First, a MUD-SIC receiver is adopted at the DN in both the SUDaided and MUD-SIC-aided systems. The per-user PER performance of a multiuser uplink system using a MUD-SIC receiver at the DN was characterized in [16] in the absence of relaying, where the uplink rate of each user was assumed to be identical. More specifically, the PER of each user is presented in [16] using the optimal decoding order, where the signal having the highest SNR is decoded, re-encoded, remodulated, and subtracted in each decoding iteration. A tight upper bound of the per-user PER is deduced in (28) of [16]. In our system, we focus our attention only on the PER performance of the desired user. We set the index of the SN to 0 , and $P_{e}^{1}$ is given in (1), where $\bar{\gamma}_{0}$ represents the average SNR of the SN at the DN, whereas $\bar{\gamma}_{1}, \ldots \bar{\gamma}_{M}$ represents the average interference-to-noise power ratio (INR) of the $M$ interferers at the $\mathrm{DN}$, respectively

$$
\begin{aligned}
P_{e}^{1} & =P_{e}^{\mathrm{MUD}-\mathrm{SIC}}\left(\bar{\gamma}_{0}, \bar{\gamma}_{1}, \ldots \bar{\gamma}_{M}\right) \\
& \leq \frac{1}{(M+1) !} \sum_{\pi}\left[\sum_{i=0}^{\pi^{-1(0)}}\left(1-\sum_{v=j}^{M} A_{v} e^{\frac{-\gamma_{\mathrm{th}}}{\lambda_{v}}}\right)\right] \prod_{i=0}^{M} \frac{1}{\phi_{i} \bar{\gamma}_{i}}
\end{aligned}
$$

\footnotetext{
${ }^{4}$ Please note that this is for brevity of outage analysis and to highlight the gist of BED-ORS proposed. If maximum-ratio combining is invoked, the expression in (2) and the correspondent BED-ORS should be modified to exploit the information in erroneous receptions. This is left for future works because of the limited space.
} 
The definitions of the parameters used are defined as follows: $\phi_{i}=\left(\sum_{u=1}^{i} \bar{\gamma}_{u}^{-1} / i\right), \quad \bar{\lambda}_{v}=\left(1-(v-j) \gamma_{\mathrm{th}}\right) /\left(v \phi_{v}\right), \quad$ and $A_{v}=$ $\prod_{u=j, u \neq v}^{n}\left(\bar{\lambda}_{v} /\left(\bar{\lambda}_{v}-\bar{\lambda}_{u}\right)\right)$.

Explicitly, $\pi$ represents the possible decoding order of the SIC, whereas $\pi(i)=0$ indicates that the desired signal is decoded during the $i$ th decoding iteration. ${ }^{5}$ Therefore, in the presence of $M$ interferers, there are $(M+1)$ ! possible decoding orders. Owning to lack of space, in this compact letter, we are unable to elaborate further on the detailed deduction of (1), which may be found in [16].

On the other hand, the PER of the retransmission phase $P_{e}^{2}$ may be expressed as

$$
P_{e}^{2}=\sum_{D_{s}} \operatorname{Pr}\left\{D_{s}\right\} P_{e}\left(D_{s}, \bar{\gamma}_{1}, \ldots, \bar{\gamma}_{M}\right)
$$

where $\operatorname{Pr}\left\{D_{s}\right\}$ is the probability that $D_{s}$ is the decoding set in the broadcast phase, which may be formulated as $\operatorname{Pr}\left\{D_{s}\right\}=\prod_{i \in D_{s}}(1-$ $\left.P_{e, s i}\right) \prod_{j \notin D s} P_{e, s j}$, where $i$ and $P_{e, s i}$ denote the $i$ th node in the decoding set and the error probability of its reception during the SN's broadcast phase, respectively. In this paper, the SUD-aided system treats the interference as noise at the RNs, and the corresponding $P_{e, s i}$ is given by [17]. The $P_{e, s i}$ of the MUD-SIC-aided system is in the form of (1), whereas the SNR and the INRs should be replaced by the values at the $i$ th RN. Therefore, the $\operatorname{Pr}\left\{D_{s}\right\}$ in (2) depends only on SN-RN channel quality and the receiver adopted at the RNs, namely, SUD or MUD-SIC receivers. Therefore, $\operatorname{Pr}\left\{D_{s}\right\}$ does not depend on the ORS design. On the contrary, the expression of $P_{e}\left(D_{s}, \bar{\gamma}_{1}, \ldots, \bar{\gamma}_{M}\right)$ in (2) depends on the ORS design, where $D_{s}$ is the decoding set and $\bar{\gamma}_{1}, \ldots, \bar{\gamma}_{M}$ represent the average INR of the $M$ interferers at the DN.

An outage-optimal ORS scheme achieves an outage probability $P_{e}^{\text {opt }}\left(D_{s}, \bar{\gamma}_{1}, \ldots, \bar{\gamma}_{M}\right)$, which is equal to the probability that transmissions from all the nodes in the decoding set $D_{s}$ to the DN failed [3]. However, $P_{e}^{\mathrm{opt}}\left(D_{s}, \bar{\gamma}_{1}, \ldots, \bar{\gamma}_{M}\right)$ cannot be simply decomposed into the form of $\prod_{i \in D_{s}} P_{e, i d}\left(\bar{\gamma}_{\mathrm{id}}, \bar{\gamma}_{1}, \ldots, \bar{\gamma}_{M}\right)$ as adopted in [3], where $P_{e, i d}\left(\bar{\gamma}_{\mathrm{id}}, \bar{\gamma}_{1}, \ldots, \bar{\gamma}_{M}\right)$ denotes the error probability at the DN when the $i$ th node of the decoding set is selected for transmission. This is because, although the ORS may rely on mutually independent SNRs as determined by $\bar{\gamma}_{\text {id }}$ and the independent fading of the current packet, the INRs represented by $\gamma_{1}, \ldots, \gamma_{M}$ are fixed for the fading realizations of the current packet, regardless of which node is selected from the decoding set. Therefore, this results in correlated error probabilities $P_{e, i d}\left(\bar{\gamma}_{\text {id }}, \bar{\gamma}_{1}, \ldots, \bar{\gamma}_{M}\right)$ for each node in the decoding set, when the MUD-SIC receivers are adopted at the DN. Therefore, it remains an open challenge to deduce the closed-form expression of $P_{e}^{\text {opt }}\left(D_{s}, \bar{\gamma}_{1}, \ldots, \bar{\gamma}_{M}\right)$, which is left for our future investigation. Instead, the focus of this paper is to conceive a beneficial ORS scheme, which achieves the optimal outage probability, when the MUD-SIC receiver is adopted at the $\mathrm{DN}$.

2) BED-ORS Design: The design criterion of ORS is to achieve the optimal $P_{e}^{\mathrm{opt}}\left(D_{s}, \gamma_{1}, \ldots, \gamma_{M}\right)$, which denotes the probability that transmission from all the nodes in $D_{s}$ to the DN failed. Therefore, an outage-optimal ORS should satisfy the following condition: If the specific retransmission by the node selected from set $D_{s}$ cannot be successfully decoded at the DN, then a retransmission by any other node picked from set $D_{s}$ would not be decoded successfully either. ${ }^{6}$

In the presence of multiple interferers at DN and when the SUD decoder is employed at the $\mathrm{DN}$, the optimal relay selection scheme is

\footnotetext{
${ }^{5}$ Note that the first iteration is indexed by 0 .

${ }^{6}$ It should be noted that, although we use the terminology of relay selection, the SN itself may be selected for retransmission as well, if no RNs decoded the message successfully during the broadcast phase or if the SN's retransmission may facilitate a better decoding result at DN compared with the RNs.
}

the same as that of the interference-free scenario [3] because the ORS scheme simply appoints the node having the highest instantaneous SNR at the DN [8]. This results in the highest instantaneous received SINR $Y_{\text {raw }}=\left(\gamma_{\mathrm{id}} /\left(\sum_{j=1}^{M} \gamma_{j}\right)\right)$, which is denoted as raw SINR at the $\mathrm{DN}$ and therefore achieves the lowest outage probability. However, if the more powerful MUD-SIC receiver is employed at the DN, the outage probability performance of relay selection is no longer determined by the raw SINR. This is due to the fact that some interfering signals may be decoded and removed from the composite signal, and hence, the effective SINR of the desired signal may be iteratively enhanced and expressed as $Y_{\text {eff }}=\left(\gamma_{\mathrm{id}} /\left(\sum_{j=k+1}^{M} \gamma_{j}\right)\right)$, where $k$ indicates that the desired signal is decoded in the $k$ th iteration, given that the previous $(k-1)$ interfering signals are successfully decoded. The SIC scheme relies on the power-based ranking of all-received signal components, and it is at its best when the received power values are clearly distinguishable. Therefore, appointing the node having the highest SNR at the DN for retransmission may be detrimental because the MUD-SIC receiver of the DN cannot reliably detect and decode the desired signal or the interference. Instead, we propose the BEDORS scheme based on the best effort detection criterion, which is implemented in Algorithm 1.

The philosophy of the BED-ORS is as follows. Retransmission by a node in decoding set $D_{s}$ may result in one of two decoding outcomes at the DN, i.e., success or failure. If the DN has the perfect knowledge of CSI for the links between each node in the decoding set and the $\mathrm{DN}$, as well as that of the links between the interferers and the DN, it may predict the decoding outcome of every node in the decoding set by implementing SIC testing, as described in Algorithm 1. Hence, the node that facilitates successful decoding at the DN may be selected.

\section{Algorithm 1: Best Effort Detection Based ORS (BED-ORS)}

1) INR ordering: Following the broadcast phase, we assume that $(L-1)$ RNs successfully decode the SN's message. Hence, there are $L$ candidate nodes in $D_{s}$, including the $\mathrm{SN}$. We denote the decoding set $D_{s}$ as $\left\{c_{1}, c_{2}, \ldots, c_{L}\right\}$. The $\mathrm{DN}$ ranks the received INR of the $M$ interfering signals $\left\{\gamma_{1}, \gamma_{2}\right.$, $\left.\ldots, \gamma_{M}\right\}$ in descending order, and the reordered INR queue is denoted as $\left\{\gamma_{(1)}, \gamma_{(2)}, \ldots, \gamma_{(M)}\right\}$, where $\gamma_{(1)} \geq \gamma_{(2)} \geq \ldots$ $\geq \gamma_{(M)}, \forall i \in[1, M]$. Then, for each node in $D_{s}$, the DN carries out SNR ordering and SIC testing, as described next.

2) SNR ordering: The DN selects a candidate node $c_{l}$ from $D_{s}$ and inserts its received SNR $\gamma_{c_{l}}$ in the INR queue, keeping the new queue, which is referred to as SIC queue $\gamma_{(1)} \geq$ $\gamma_{(2)} \geq \cdots \geq \gamma_{(M+1)}$ in a descending order. This is the optimal decoding order for equal-rate scenario, which is proved in [16].

3) SIC testing: First, for each position $i$ in the INR queue, an SNR upper threshold $\gamma_{i}^{u}$ and an SNR lower threshold $\gamma_{i}^{l}$ are calculated as $\gamma_{i}^{u}=\left(\gamma_{(i-1)} / \gamma_{\mathrm{th}}\right)-\sum_{j=i}^{M} \gamma_{(j)}-1$ and $\gamma_{i}^{l}=$ $\gamma_{\text {th }}\left(\sum_{j=i}^{M} \gamma_{(j)}+1\right)$ for the SIC testing step, where $\gamma_{\text {th }}$ is the predefined threshold to be exceeded for successful decoding. The bounds are derived as follows: If the SNR of a candidate node is inserted at position $i$, to facilitate successful decoding, a necessary condition is that we have $\left(\gamma_{(i-1)} /\left(\gamma_{c_{l}}+\right.\right.$ $\left.\left.\sum_{j=i}^{M} \gamma_{(j)}+1\right)\right) \geq \gamma_{\mathrm{th}}$ and $\left(\gamma_{c_{l}} /\left(\sum_{j=i}^{M} \gamma_{(j)}+1\right)\right) \geq \gamma_{\mathrm{th}}$, where the first inequality ensures that the SINR of the signal at position $(i-1)$ exceeds $\gamma_{\mathrm{th}}$; therefore, it is decoded successfully. By contrast, the second inequality ensures that the SINR of the candidate's signal exceeds $\gamma_{\mathrm{th}}$. Please note that the condition is not a sufficient condition because it also relies on the decoding outcomes of the preceding signals in the SIC queue. 


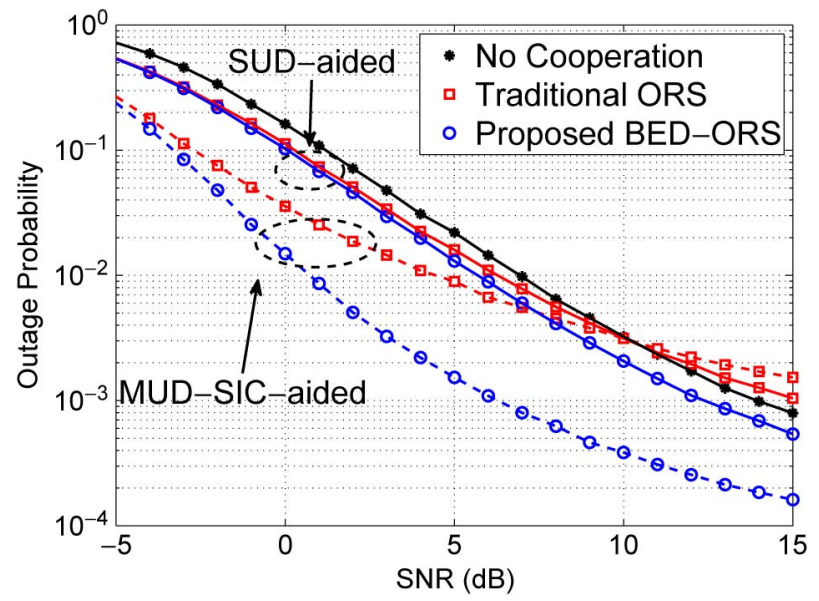

Fig. 2. Outage probability of different schemes versus the SN-DN link SNR for transmissions over block Rayleigh fading channels for $S I R=0 \mathrm{~dB}$, $\boldsymbol{R}_{T}=0.5 \mathrm{bit} / \mathrm{s} / \mathbf{H z}$, two RNs and four INs. The traditional ORS scheme is based on the highest SNR criteria, whereas the proposed BED-ORS scheme is implemented by Algorithm 1 assuming perfect CSI.

Denoting that after SNR ordering, $\gamma_{c_{l}}$ is inserted at position $k$. A failure is declared when the SINR of the signal in the $i$ th $(i<k)$ decoding iteration does not exceed $\gamma_{\mathrm{th}}$; therefore, none of the signals following in the queue (including the candidate signal) can be decoded because their SINR is even lower. Otherwise, if each $i$ th signal $(i \leq k)$ in the SIC queue is successfully decoded and $\gamma_{k}^{u} \geq \gamma_{c_{l}} \geq \gamma_{k}^{l}$ is satisfied, a successfully decoding outcome may be declared.

4) Node selection: If the decoding outcome predicted for $c_{l}$ is a success, $c_{l}$ is selected as the transmitting node and the node's index is sent by the DN through a feedback channel to the wouldbe-transmit node. Otherwise, the DN selects another candidate node that has not been tested yet and repeats steps 2 and 3. If decoding outcome predicted for all nodes in $D_{s}$ is a failure, an outage event is declared.

The proposed BED-ORS tests all candidates in $D_{s}$ until the decoding outcome of a candidate is successful and hence selected, or no candidates output a successful decoding outcome. Therefore, it is outage optimal by ensuring that if the node selected cannot facilitate successful decoding, any other nodes in $D_{s}$ would not be decoded successfully either. It should be noted that the proposed BEDORS does not involve the MUD-SIC decoding process. The DN may utilize MUD-SIC to decode the bits or symbols at the physical layer to recover the information transmitted. However, the BED-ORS is a MAC layer algorithm, which imitates the MUD-SIC process and relies only on processing of the SNRs and INRs. From the physical layer's perspective, the channel state information of the channels between all RNs and the DN and those of the channels between all INs and the DN are required by the MUD-SIC detecting stage. However, the physical layer SNRs and INRs have to be fed into the MAC layer and then exploited at the ORS stage, as it will be shown later in the simulation results in Fig. 2.

To support SIC decoding at the physical layer, the MAC layer of the ORS scheme originally proposed in [3] should be redesigned. The DN has the knowledge of the interference power; hence, it may serve as the central controller, which requires the CSI of the channels spanning from all candidate RNs to the DN. Therefore, we may summarize the MAC layer design conceived for our BED-ORS as follows. After the $\mathrm{SN}$ and DN communicate by exchanging their request-to-send and clear-to-send messages, then a NACK message is sent by the DN if the $\mathrm{DN}$ does not decode the data successfully. If an $\mathrm{RN}$ decodes the data, it sends a relay-request-to-send (RRTS) message and the DN hence becomes capable of estimating the channel spanning from all candidate RNs to the DN. Then, the relay selection is carried out by using Algorithm 1. Finally, the DN sends a relay-clear-to-send message, which includes the selected RN's identity.

Since in the MAC layer protocol proposed in [3], no interference is considered, the RNs contend to access the channel by setting up distributed timers at the RNs, where the RN having the highest SNR at the DN may be selected. Therefore, the contention period is independent of the number of RNs equipped with back-off timers. However, when there is CCI at the DN and the MUD-SIC is adopted, a practical issue arises from the fact that the CSI of the channels spanning from all candidate RNs to the DN has to be known at the DN. Therefore, each RN has to send a RRTS message to the DN for CSI estimation; hence, the total period required for the RRTS messaging is proportional to the number of candidate RNs. Therefore, the number of participating RNs should be appropriately controlled to avoid exceedingly high overall delays during the relay selection phase.

3) Complexity Analysis: Compared with the traditional ORS scheme based on the highest received SNR/SINR [3], [8], the proposed BED-ORS imposes higher complexity at the DN. Assuming $K \mathrm{RNs}$ and $M$ INs, the worst case in terms of the ORS implementation complexity is that the decoding set includes $(K+1)$ candidates in $D_{s}$, where all RNs decode successfully after the broadcast phase. Considering the merge sorting algorithm [18], the INR ordering of Algorithm 1 requires approximately $M \log _{2} M-M+1$ comparison operations, whereas the associated threshold calculation requires $2(M+1)$ division operations and $(M-1)$ addition operations. The worst case complexity of the SNR-based ordering requires $(K+1) \log _{2} M$ comparison operations to insert a candidate's SNR into the INR queue $(K+1)$ times. $^{7}$ SIC testing requires two comparison operations. Therefore, the overall number of computations for Algorithm 1's worst case scenario are $(K+M+1) \cdot \log _{2} M-M+2(K+1)+1$ comparison operations, $2(M-1)$ additions, and $2(M+1)$ divisions. In comparison, the traditional ORS scheme [3], [8] requires at worst $K$ comparisons to select the node from $D_{s}$ having the highest SNR. For example, for $K=M=4$, the numbers of comparison, addition, and division operations required by Algorithm 1 in the worst case are approximately 25,6 , and 10 , respectively, whereas the traditional ORS scheme requires at worst four comparison operations.

It should be noted that, although the BED-ORS introduces higher implementation complexity, it is negligible compared with decoding complexity imposed by the decoding process at the physical layer. For example, a state-of-art low-complexity design of a turbo decoder [19] requires multiple add-compare-select (ACS) operations per bit per iteration. Assuming a frame length of 1000 bits, a turbo decoder using four ACS operations, and four iterations, then it requires 8000 ACS operations for decoding a frame. In contrast, as BED-ORS is based on a frame-by-frame mechanism, it requires 25 comparison, 6 addition, and 10 division operations for a frame, assuming a scenario of four RNs and four INs. Therefore, the BED-ORS involves much lower complexity than the decoding process. We considered the classic cellular uplink scenario. If fixed RNs are installed by the operators, the RNs may indeed have sufficient processing capability to adopt MUD-SIC receivers. On the other hand, if user cooperation is adopted, where the RNs are mobile users, our SUD receivers may be adopted at the RN. Although the proposed ORS scheme imposed increased implementation complexity, it may exhibit significant outage probability improvements, as evidenced in Section V, where the implementation issues are discussed in more detail.

${ }^{7}$ All $K$ RNs successfully decode the SN's signal and join $D_{s}$; therefore, the size of $D_{s}$ is $(K+1)$. 


\section{OPTIMIZATION FOR OUTDATED CSI}

In the previous discussions, perfect CSI was assumed available at the RNs and the DN in the BED-ORS scheme. However, if the channel's coherence time is shorter than the time required for processing the relay selection plus the delay of feeding back the selected node index by the DN, the CSI used for ORS may become outdated and hence may be different from the actual CSI. As a result, the accuracy of the predictions may degrade. In this case, if the predicted decoding outcomes of multiple nodes in the decoding sets are a success, it is optimal to select the outcome where the probability that the actual decoding outcome being a success is maximized. Therefore, it should be emphasized that with outdated CSI, the ORS scheme is not guaranteed to be outage optimal. Alternatively, we may design an ORS scheme that maximizes the probability of avoiding outage or minimizes the probability of outage [15]. Therefore, we define the probability of avoiding an outage of candidate $c_{l}$ as the probability that the actual decoding outcome is a success, conditioned on the prediction being a success, denoted as $P_{f}^{c_{l}}$. The candidate $c_{\star}$ having the highest probability of avoiding an outage is selected as follows: $c_{\star}=\arg \max _{c_{l} \in D_{s}}\left\{P_{f}^{c_{l}}\right\}$.

To acquire $P_{f}^{c_{l}}$ for each $c_{l} \in D_{s}$, we have to evaluate the impact of outdated CSI. Using a similar approach as in [12], the correlation coefficient between the actual channel coefficient $h$ and the outdated channel coefficient $\hat{h}$ is defined as $\rho$. Therefore, we have [12]: $h=$ $\rho \hat{h}+\sqrt{1-\rho^{2}} v$, where $v \sim C N(0,1)$ and $\rho$ is assumed to be a constant in this paper. The actual SNR $\gamma$ conditioned on its estimate $\hat{\gamma}$ follows a noncentral chi square distribution with two degrees of freedom, and the probability density function of the SNR is expressed as [12]:

$$
f_{\gamma \mid \hat{\gamma}}(\gamma \mid \hat{\gamma})=\frac{1}{\bar{\gamma}\left(1-\rho^{2}\right)} e^{-\frac{\gamma+\rho^{2} \hat{\gamma}}{\bar{\gamma}\left(1-\rho^{2}\right)}} I_{0}\left(\frac{2 \rho \sqrt{\gamma \hat{\gamma}}}{\bar{\gamma}\left(1-\rho^{2}\right)}\right)
$$

where $\bar{\gamma}$ denotes the average $\mathrm{SNR}$, and $I_{0}(\cdot)$ is the zero-order modified Bessel function of the first kind. In our analysis, we assume that the CSIs of the candidates in the decoding set are outdated, whereas the CSIs of the interferers are perfect. Therefore, $\gamma_{i}^{l}$ and $\gamma_{i}^{u}$ defined in Step 1 of Algorithm 1 are not outdated. Then, the candidate $c_{l}$ 's probability of avoiding an outage $P_{f}^{c_{l}}$ may be expressed as [20]

$$
\begin{aligned}
P_{f}^{c_{l}} & =\operatorname{Pr}\left\{\gamma_{k}^{l} \leq \gamma_{c_{l}} \mid \hat{\gamma}_{c_{l}}\right\} \operatorname{Pr}\left\{\gamma_{c_{l}} \leq \gamma_{k}^{u} \mid \hat{\gamma}_{c_{l}}\right\} \\
\operatorname{Pr}\left\{\gamma_{c_{l}} \leq \gamma_{k}^{u} \mid \hat{\gamma}_{c_{l}}\right\} & =\int_{0}^{\gamma_{k}^{u}} f_{\gamma_{c_{l}} \mid \hat{\gamma}_{c_{l}}}\left(\gamma_{c_{l}} \mid \hat{\gamma}_{c_{l}}\right) d \gamma_{c_{l}} \\
& =1-Q_{1}\left(\lambda, b_{u}\right) \\
\operatorname{Pr}\left\{\gamma_{k}^{l} \leq \gamma_{c_{l}} \mid \hat{\gamma}_{c_{l}}\right\} & =1-\int_{0}^{\gamma_{k}^{l}} f_{\gamma_{c_{l}} \mid \hat{\gamma}_{c_{l}}}\left(\gamma_{c_{l}} \mid \hat{\gamma}_{c_{l}}\right) d \gamma_{c_{l}} \\
& =Q_{1}\left(\lambda, b_{l}\right)
\end{aligned}
$$

where $Q_{1}(\lambda, b)$ is the Marcum Q-function of order 1, whereas $\lambda, b_{u}$ and $b_{l}$ are defined as [20]: $\lambda=\rho \sqrt{\left(2 \hat{\gamma}_{c_{l}} /\left(\bar{\gamma}_{c_{l}}\left(1-\rho^{2}\right)\right)\right)}$, $b_{u}=\left(2 \gamma_{k}^{u} /\left(\bar{\gamma}_{c_{l}}\left(1-\rho^{2}\right)\right)\right)^{2}$, and $b_{l}=\left(2 \gamma_{k}^{l} /\left(\bar{\gamma}_{c_{l}}\left(1-\rho^{2}\right)\right)\right)^{2}$. Hence, to achieve the lowest outage probability, Algorithm 1 may be revised as follows:

\section{Algorithm 2: I-BED-ORS using outdated-CSI}

1) INR ordering: The same procedure of INR ordering is implemented as in Algorithm 1.

2) SNR ordering: The DN selects a candidate node $c_{l}$ and the same procedure of SNR ordering is implemented as in Algorithm 2.
3) SIC testing: The same procedure of SIC testing is implemented as in Algorithm 2. Additionally, the probability of avoiding an outage $P_{f}^{c_{l}}$ is calculated using (4)-(6).

4) Node selection: For every candidate node $c_{l}$ in $D_{s}$, the DN estimates the decoding outcomes and selects the node $c_{l^{\star}}$ of highest probability of avoiding an outage obeying $c_{l^{\star}}=$ $\max _{c_{l} \in D_{s}} P_{f}^{c_{l}}$. Then, this node's index is sent by the DN to the relevant $\mathrm{RN}$ via a feedback channel.

As shown in (4)-(6), the calculation of probability of avoiding an outage $P_{f}^{c_{l}}$ involves integration and may be computational expensive. As $\rho$ and $\bar{\gamma}_{c_{l}}$ are statistical parameters, which are assumed to be known so that $\lambda$ is known at the DN, a lookup table connecting the values of $Q_{1}(\lambda, b)$ and $b$ may facilitate acceptable implementation complexity. ${ }^{8}$

\section{Simulation Results and Discussions}

Here, we evaluate the performance of the two proposed systems in terms of the associated packet loss ratio (outage probability). In all our simulations, we consider a topology comprising $K$ RNs, all placed halfway along the line between the SN and DN, and we set the channel's path loss exponent to 3 . Additionally, we assume that all nodes in the network use an identical transmit power value. The time-average SNR of the SN-DN channel is denoted as $\bar{\gamma}_{s, d}$. Therefore, the SNR of the channel between any two nodes is $\bar{\gamma}_{i, j}=\left(d_{i, j} / d_{s, d}\right)^{-\alpha} \bar{\gamma}_{s, d}$, where $d_{i, j}$ denotes the distance between node $i$ and node $j$ and $\alpha$ is the channel's path loss exponent. The channels are assumed to be subjected to independent Rayleigh block fading. Throughout the simulations, the transmission rate of each $\mathrm{SN}$ is $R_{T}=0.5 \mathrm{bit} / \mathrm{s} / \mathrm{Hz}$.

4) Effects of ORS Criteria: We compare the outage probability performance of the traditional ORS scheme based on the highest received SNR/SINR criterion to that of the proposed BED-ORS scheme based on the best effort detection criterion described in Algorithm 1. It is also assumed that the average signal-to-interference power ratio (SIR) for each IN is $0 \mathrm{~dB} .{ }^{9}$ The number of RNs and INs is two and four, respectively. Fig. 2 shows the outage probability of the proposed MUD-SIC-aided CARQ system and of the SUD-aided CARQ system. The traditional ORS in Fig. 2 indicates a simple combination of the MUD-SIC with ORS relying on [3], which does not exploit the INRs during the relay selection process. When the traditional ORS scheme based on the highest received $S N R$ criterion is used, it is observed that in Fig. 2, a small improvement in outage probability performance is achieved by the MUD-SIC-aided CARQ system and SUD-aided CARQ system over the noncooperative system when the SN-DN SNR is within the range from $-5 \mathrm{~dB}$ to $-10 \mathrm{~dB}$. However, this trend no longer holds for higher SN-DN SNRs, where the employment of cooperation seems to be detrimental in terms of the outage probability performance, as shown in Fig. 2.

The reason for this behavior was provided in Section III. In this case, selecting an RN having a lower SNR at the DN may be preferred, where the strong interfering signals may be successfully decoded and subtracted, resulting in an improved SINR for the desired signal and facilitating a higher probability of successful decoding, which is the philosophy behind the BED-ORS scheme. Fig. 2 shows that both the MUD-SIC-aided and SUD-aided systems using BED-ORS scheme

\footnotetext{
${ }^{8}$ More practically, even if $\rho$ and $\bar{\gamma}_{c_{l}}$ are unknown at the DN, a heuristic method may be implemented by selecting $c_{l^{\star}}=\max _{c_{l} \in D_{s}} \min \left(\gamma_{k}^{u}-\right.$ $\left.\gamma_{c_{l}}, \gamma_{c_{l}}-\gamma_{k}^{l}\right)$.

${ }^{9}$ The average SIR is measured at the RNs in the broadcast phase, whereas at the DN, the SIR in the broadcast phase for current setting is approximately $-9 \mathrm{~dB}$. For simplicity, we use SIR at RNs in the broadcast phase to measure interference power.
} 


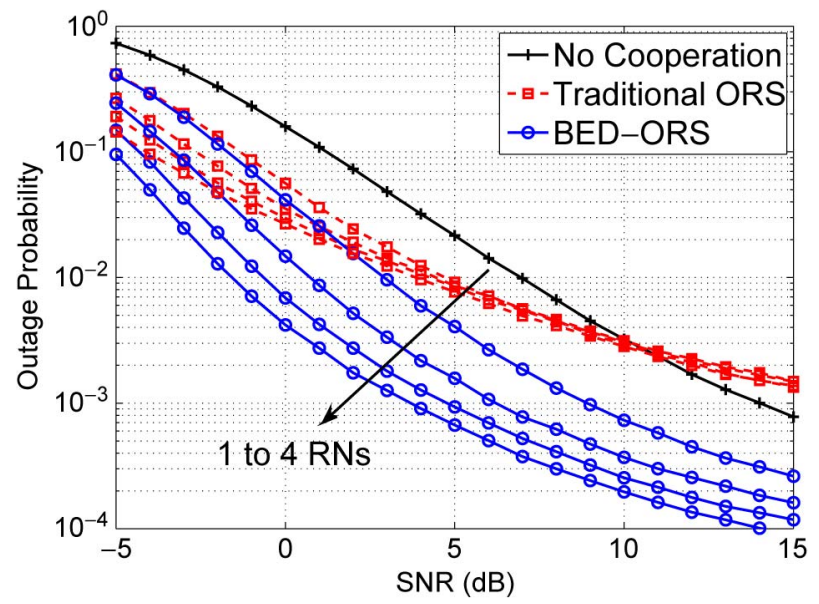

Fig. 3. Outage probability versus the SN-DN link SNR for transmissions over block Rayleigh fading channels with SIR $=0 \mathrm{~dB}, R_{T}=0.5 \mathrm{bit} / \mathrm{s} / \mathrm{Hz}$, four INs, and one to four RNs. The MUD-SIC-aided scheme is adopted. The traditional ORS scheme is based on the highest SNR criteria, whereas the proposed BEDORS scheme is implemented by Algorithm 1 assuming perfect CSI.

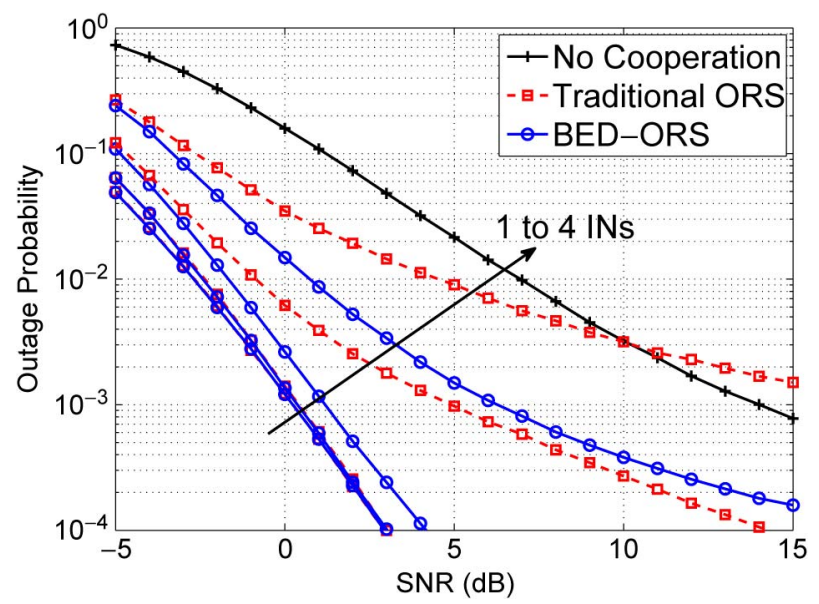

Fig. 4. Outage probability versus the SN-DN link SNR for transmissions over block Rayleigh fading channels with $\mathrm{SIR}=0 \mathrm{~dB}, R_{T}=0.5 \mathrm{bit} / \mathrm{s} / \mathrm{Hz}$, two RNs, and one to four INs. The MUD-SIC-aided scheme is adopted. The traditional ORS scheme is based on the highest SNR criteria, whereas the proposed BED-ORS scheme is implemented by Algorithm 1 assuming perfect CSI.

implemented in Algorithm 1 outperform the noncooperative system for all the SNRs considered. At $P_{l}=10^{-3}$, a substantial gain of about $7.5 \mathrm{~dB}$ is achieved by the MUD-SIC-aided system relying on two $\mathrm{RNs}$ over the noncooperative system, whereas only a $1.8-\mathrm{dB}$ gain is achieved by the SUD-aided system.

5) Effects of Number of Relays, Interferer, and Interference Power: Then, we evaluate the performance of our proposed systems for different numbers of RNs and INs. First, the number of INs is fixed to four, and the number of RNs is increased from one to four. It is observed in Fig. 3 that, as the number of RNs increases, the outage performance of the MUD-SIC-aided system adopting the proposed BED-ORS scheme was gradually improved as a benefit of increased spatial diversity, which outperforms the traditional ORS scheme.

Then, the number of RNs is fixed to two, and the number of INs increases from one to four. It is observed in Fig. 4 that the proposed BED-ORS scheme always achieves better outage performance than the traditional ORS for different number of INs. However, when the number of INs is one or two, the outage performance of traditional

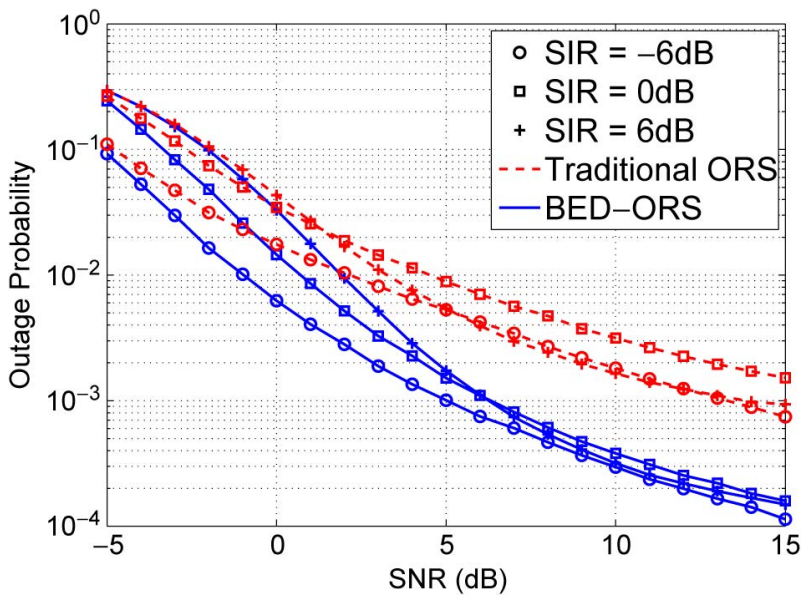

Fig. 5. Outage probability versus the SN-DN link SNR for transmissions over block Rayleigh fading channels with $S I R=-6,0$, and $6 \mathrm{~dB} ; R_{T}=$ $0.5 \mathrm{bit} / \mathrm{s} / \mathrm{Hz}$; two RNs; and four INs. The MUD-SIC-aided scheme is adopted. The traditional ORS scheme is based on the highest SNR criteria, whereas the proposed BED-ORS scheme is implemented by Algorithm 1 assuming perfect CSI.

ORS is very close to that of BED-ORS. The reason is that when the number of INs is relatively small and the resultant SINR is sufficiently high, the performance of a MUD-SIC receiver and a SUD receiver is identical.

The effects of interference power are evaluated in Fig. 5, where an $\mathrm{SN}$ assisted by two RNs and four INs is considered. We consider three signal-to-interference power levels, i.e., $S I R=0,6$, and $12 \mathrm{~dB}$, respectively. The dashed lines represent the performance of traditional ORS, whereas the solid lines represent the performance of the BEDORS under different SIR levels. It is observed that the BED-ORS scheme achieves better outage performance than traditional ORS. It is also observed that at low SNR region, a higher SIR may result in worse outage performance. The reason is that the probability of the interference being successfully decoded and removed is low due to the low INR. Assuming a constant SIR, as SNR increases, the INR correspondingly increases and therefore achieving higher probability of decoding and removing interference, which in turn results in improved outage performance.

On the other hand, Fig. 6 shows the importance of employing the more powerful MUD-SIC receiver at the DN in the presence of interference. If the DN adopts SUD receivers, the PLR performance improvement achieved by enhancing SN-DN link quality erodes at moderate SNRs and the system encounters the interference-limited region, as shown by the dotted lines. The proposed SUD- and MUDSIC-aided schemes, which are represented by the dashed and solid lines, respectively, are capable of achieving better outage performance, with the aid of MUD-SIC receivers at the DN. We may conclude that in the presence of interference, a DN adopting the MUD-SIC receiver is the guarantee of achieving a low outage probability. As the SUD receivers exhibit lower implementation decoding complexity than the MUD-SIC receivers, SUD-aided scheme may be preferred because of low-complexity RNs, provided that the outage performance degradation remains tolerable. Fig. 5 shows an example associated with $S I R=12 \mathrm{~dB}$, where the degradation of the SUD-aided scheme is approximately $1.2 \mathrm{~dB}$ at $P L R=10^{-3}$ compared with the MUDSIC-aided scheme.

Practically, the decoding complexity of the MUD-SIC increases at most linearly with the number of interferers. Therefore, we are capable of controlling the number of interferers actually decoded by the MUDSIC receivers. In other words, we may process a limited number 


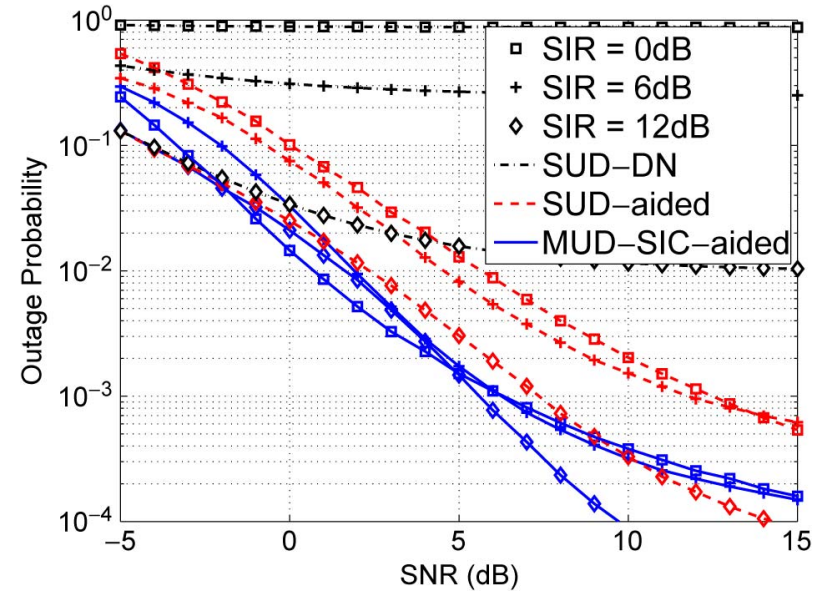

Fig. 6. Outage probability versus the SN-DN link SNR for transmissions over block Rayleigh fading channels with $S I R=0,6$, and $12 \mathrm{~dB} ; R_{T}=$ $0.5 \mathrm{bit} / \mathrm{s} / \mathrm{Hz}$; two RNs; and four INs. The MUD-SIC-aided and SUDsided schemes are compared. The BED-ORS scheme is implemented by Algorithm 1 assuming perfect CSI.

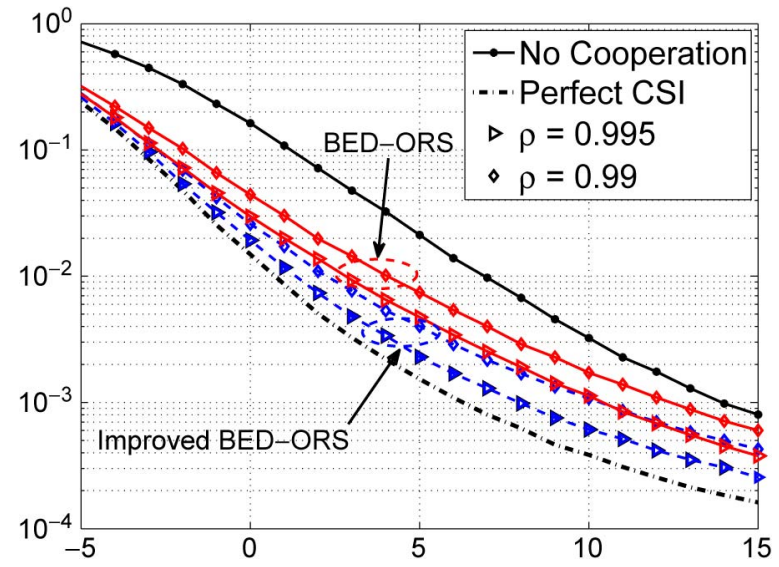

Fig. 7. Outage probability versus the SN-DN link SNR for transmissions over block Rayleigh fading channels with outdated CSI, where correlation coefficient $\rho=0.99,0.995$. SIR $=0 \mathrm{~dB}, R_{T}=0.5 \mathrm{bit} / \mathrm{s} / \mathrm{Hz}$, two RNs, and four INs. The BED-ORS is implemented by Algorithm 1, and I-BED-ORS is implemented by Algorithm 2. Only the performance of the MUD-SIC-aided CARQ is shown because it exhibits highest outage improvements toward the noncooperative counterparts.

of strong interferers using MUD-SIC while treating the remaining interferers as noise. In this way, we may remove the most detrimental components and strike a balance between the outage performance achieved and the complexity imposed.

6) Effects of Outdated CSI and Using I-BED-ORS: Fig. 7 shows the outage probability performance of the ORS proposed in Section IV. If the CSI is outdated and the DN employs the suboptimum Algorithm 1 based on perfect CSI, the outage probability performance may severely degrade, as shown in Fig. 7. By contrast, when the I-BED-ORS implemented in Algorithm 2 is used, the outage probability performance may be substantially improved, as shown in Fig. 7. However, as the correlation coefficient $\rho$ decreases and the reliability of the outdated CSI drops, the outage probability performance of the optimized ORS using Algorithm 2 degrades. It may be concluded that the accuracy of CSI used for ORS is critical for the achievable outage probability performance of cooperative systems adopting MUD-SIC receivers.

\section{CONCLUSION}

In this paper, we have considered two CARQ systems adopting MUD-SIC receivers at the DN, namely, the MUD-SIC-aided CARQ and SUD-aided CARQ schemes. The outage-optimal BED-ORS was proposed for both systems based on a best effort detection criterion. The proposed systems were shown to outperform both the noncooperative system and the system employing SUD receivers at the DN, whereas the MUD-SIC-aided system achieved significant outage probability performance improvements with the aid of the BEDORS. Additionally, an optimized BED-ORS scheme was designed, which was shown to have improved robustness against degraded CSI accuracy imposed by outdated CSI.

\section{REFERENCES}

[1] B. Zhao and M. Valenti, "Practical relay networks: A generalization of hybrid-ARQ," IEEE J. Sel. Commun., vol. 23, no. 1, pp. 7-18, Jan. 2005.

[2] Y. Jing and H. Jafarkhani, "Single and multiple relay selection schemes and their achievable diversity orders," IEEE Trans. Wireless Commun., vol. 8, no. 3, pp. 1414-1423, Mar. 2009.

[3] A. Bletsas, H. Shin, and Z. Win, "Cooperative communications with outage-optimal opportunistic relaying," IEEE Trans. Wireless Commun., vol. 6, no. 9, pp. 3450-3460, Sep. 2007.

[4] I. Krikidis, T. Charalambous, and J. S. Thompson, "Buffer-aided relay selection for cooperative diversity systems without delay constraints," IEEE Trans. Wireless Commun., vol. 11, no. 5, pp. 1957-1967, May 2012.

[5] F. Etezadi, K. Zarifi, A. Ghrayeb, and S. Affes, "Decentralized relay selection schemes in uniformly distributed wireless sensor networks," IEEE Trans. Wireless Commun., vol. 11, no. 3, pp. 938-951, Mar. 2012.

[6] C.-H. Yu and O. Tirkkonen, "Opportunistic multiple relay selection with diverse mean channel gains," IEEE Trans. Wireless Commun., vol. 11, no. 3, pp. 885-891, Mar. 2012.

[7] I. Krikidis, J. Thompson, S. Mclaughlin, and N. Goertz, "Max-min relay selection for legacy amplify-and-forward systems with interference," IEEE Trans. Wireless Commun., vol. 8, no. 6, pp. 3016-3027, Jun. 2009.

[8] D. Lee and J. H. Lee, "Outage probability of decode-and-forward opportunistic relaying in a multicell environment," IEEE Trans. Veh. Technol., vol. 60, no. 4, pp. 1925-1930, May 2011.

[9] H. Yu, I.-H. Lee, and G. L. Stuber, "Outage probability of decodeand-forward cooperative relaying systems with co-channel interference,' IEEE Trans. Wireless Commun., vol. 11, no. 1, pp. 266-274, Jan. 2012.

[10] D. Tse and P. Viswanath, Fundamentals of Wireless Communication. Cambridge, U.K.: Cambridge Univ. Press, 2005.

[11] C. Gong, A. Tajer, and X. Wang, "Group decoding for multi-relay assisted interference channels," IEEE J. Sel. Areas Commun., vol. 30, no. 8, pp. 1489-1499, Sep. 2012

[12] J. Vicario, A. Bel, J. Lopez-Salcedo, and G. Seco, "Opportunistic relay selection with outdated CSI: Outage probability and diversity analysis," IEEE Trans. Wireless Commun., vol. 8, no. 6, pp. 2872-2876, Jun. 2009.

[13] M. Chen, T. C.-K. Liu, and X. Dong, "Opportunistic multiple relay selection with outdated channel state information," IEEE Trans. Veh. Technol., vol. 61, no. 3, pp. 1333-1345, Mar. 2012.

[14] S. Kim, S. Park, and D. Hong, "Performance analysis of opportunistic relaying scheme with outdated channel information," IEEE Trans. Wireless Commun., vol. 12, no. 2, pp. 538-549, Feb. 2013.

[15] Y. Li, Q. Yin, W. Xu, and H.-M. Wang, "On the design of relay selection strategies in regenerative cooperative networks with outdated CSI," IEEE Trans. Wireless Commun., vol. 10, no. 9, pp. 3086-3097, Sep. 2011.

[16] V. Lau, "Per-user packet outage analysis in slow multiaccess fading channels with successive interference cancellation for equal rate applications," IEEE Trans. Wireless Commun., vol. 7, no. 5, pp. 1754-1763, May 2008.

[17] D. Lee and J. H. Lee, "Outage probability for dual-hop relaying systems with multiple interferers over Rayleigh fading channels," IEEE Trans. Veh. Technol., vol. 60, no. 1, pp. 333-338, Jan. 2011.

[18] T. H. Cormen, C. Stein, R. L. Rivest, and C. E. Leiserson, Introduction to Algorithms., 2nd ed. New York, NY, USA: McGraw-Hill, 2001.

[19] L. Li, R. G. Maunder, B. M. Al-Hashimi, and L. Hanzo, "A lowcomplexity turbo decoder architecture for energy-efficient wireless sensor networks," IEEE Trans. Very Large Scale Integr. (VLSI) Syst., vol. 21, no. 1, pp. 14-22, Jan. 2013.

[20] A. Nuttall, "Some integrals involving the Q function," IEEE Trans. Inf. Theory, vol. 21, no. 1, pp. 95-96, Jan. 1975. 


\section{Correspondence}

\section{Opportunistic Relay Selection for Cooperative Relaying in Cochannel Interference Contaminated Networks}

\author{
Bo Zhang, Mohammed El-Hajjar, and Lajos Hanzo
}

\begin{abstract}
A multiple-relay assisted cooperative automatic-repeatrequest (CARQ) system is proposed in interference-limited scenario, where multiple-user detection (MUD) receivers using successive interference cancellation (SIC) are employed at the relay nodes (RNs) and the destination node (DN) to combat cochannel interference (CCI). We analyze the outage performance and propose a novel best effort-detection-based opportunistic relay selection (BED-ORS) scheme, which achieves significantly better outage performance than the traditional highest SNR-based ORS scheme offered in the literature. Moreover, considering the effects of the outdated channel state information (CSI), an improved-BED-ORS (I-BED-ORS) scheme is proposed to enhance the robustness against CSI imperfections.
\end{abstract}

Index Terms-Cooperative diversity, multiple access interference, opportunistic relay selection (ORS), outdated channel state information (CSI).

\section{INTRODUCTION}

Cooperative communication is capable of providing spatial diversity for single-antenna aided wireless devices [1], where one or more nodes act as relays for other communicating nodes. Hence, relay selection [2] is an important factor that affects the performance of a cooperative system. As the coordination and synchronization of multiple simultaneously transmitting relay nodes (RNs) constitutes a challenge, the opportunistic relay selection (ORS) [3] may be preferred, which achieves the maximum attainable diversity at lower implementation complexity. From multiple candidate RNs, the ORS may select a single RN that facilitates the highest instantaneous SNR at the destination node (DN) to retransmit the signal from the source node (SN) [3]. In this way, a diversity order equal to the number of RNs $K$ can be achieved [3]. ${ }^{1}$ Recently, several improved ORS algorithms for different scenarios have been proposed. The authors of [4] studied the relay selection for a finite-buffer decode-and-forward cooperative wireless network, and it is shown to achieve better outage performance than the systems without aid of buffers. In [5], the authors investigated and compared the optimal highest SNR-based selection, the geometrybased selection, and the random selection for uniformly distributed

Manuscript received March 24, 2013; revised September 19, 2013 and November 8, 2013; accepted November 11, 2013. This work was supported in part by the Research Councils U.K. under India-U.K. Advanced Technology Centre (IU-ATC), by the European Union under the Concerto project, by the European Research Council under its Advanced Fellow Scheme, and by China-U.K. Scholarship Council. The review of this paper was coordinated by Prof. J.-Y. Chouinard.

B. Zhang is with the School of Electronics and Computer Science, University of Southampton, Southampton SO17 1BJ, U.K., and also with the School of Electronics and Electrical Engineering, National University of Defense Technology, Changsha 410073, China (e-mail: bz2g10@ecs.soton.ac.uk).

M. El-Hajjar and L. Hanzo are with the School of Electronics and Computer Science, University of Southampton, Southampton SO17 1BJ, U.K. (e-mail: meh@ecs.soton.ac.uk; lh@ecs.soton.ac.uk).

Color versions of one or more of the figures in this paper are available online at http://ieeexplore.ieee.org.

Digital Object Identifier 10.1109/TVT.2013.2291162

${ }^{1}$ If the direct link between the $\mathrm{SN}$ and the DN is considered, a maximum diversity order of $(K+1)$ may be achieved. sensor networks, where they have shown that for all proposed schemes, the SNR variance at the DN converges to zero as the number of RNs increases, which effectively mitigate the effects of fading. In [6], an ORS was designed to select multiple relays rather than a single relay to achieve higher end-to-end throughput.

However, the practical networks may suffer from the cochannel interference (CCI), which was not considered in the previous works. Recently, a dual-hop cooperative relaying scheme adopting singleuser detection (SUD) receivers was investigated to mitigate CCI [7][9]. When SUD receivers are adopted at the DN, the authors of [8] show that the traditional ORS based on the highest SNR criterion is outage optimal in a dual-hop relaying system adopting the SUD receivers. However, the SUD receivers treat CCI as noise and suppress it, where the signal structure of the interference is not exploited. In contrast to SUD, multiuser detection relying on successive interference cancellation (MUD-SIC) [10] may achieve better outage performance while introducing moderate implementation complexity [10]. In [11], MUD-SIC receivers were introduced into a cooperative network with multiple SNs, RNs, and DNs, where the transmission rates were optimized for a scenario where multiple RNs access the channel simultaneously.

The conventional MUD-SIC itself is generally adopted at the detection and decoding stage as a physical layer operation to improve the outage performance of the multiuser uplink scheme or a pointto-point (P2P) scheme in the presence of interference, but it has not been intrinsically amalgamated with any ORS design or cooperative automatic-repeat-request (CARQ) scheme as a medium access control (MAC) layer protocol in the existing literature. Therefore, the first research question we consider can be stated as: How to design outageoptimal ORS using MUD-SIC receivers? Furthermore, the outage performance of ORS may degrade due to having a realistic delayed feedback, where the selection decisions are based on the outdated channel state information $(\mathrm{CSI})^{2}$ [12], [13]. However, previous research considers ORS schemes either using outdated CSI assuming interference-free scenarios [12]-[14] or assuming perfect CSI in the presence of CCI [8], [15]. Therefore, our second research question is stated as: How to mitigate the outage performance degradation under outdated CSI for the ORS scheme using MUD-SIC receivers?

Against this background, in this paper, we consider two CARQ systems employing MUD-SIC receivers at the DN. In the first system, which we refer to as $\boldsymbol{S U D}$-aided $\boldsymbol{C A R Q}$, we employ SUD receivers at the RNs. By contrast, in the second system, which we refer to as MUD-SIC-aided CARQ, we employ MUD-SIC receivers at both the DN and the RNs. ${ }^{3}$ Then, we try to address the aforementioned two open problems in the proposed system models, and the novel contributions in this paper may be summarized as follows.

- We analyze the outage performance of the proposed systems and define the criteria for an outage-optimal ORS scheme for systems adopting MUD-SIC receivers at the DN. We design a

\footnotetext{
${ }^{2}$ In this paper, the CSI is defined as the instantaneous channel coefficient capturing the effects of the path loss and fading.

${ }^{3}$ Although a single SN-DN pair is considered in our system, the ORS scheme proposed may be generalized to practical multiuser networks composed of multiple SNs, e.g., in a cellular network, where multiple SN-DN pairs in a cell may be assigned orthogonal channels so that the ORS may be implemented separately for each SN-DN pair, whereas the CCI is dominated by intercell interference.
} 


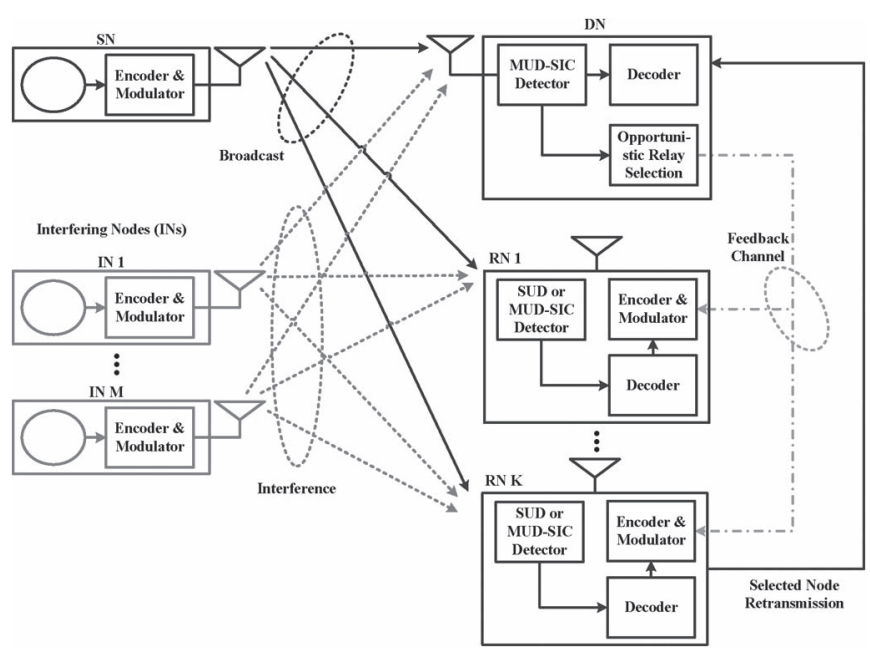

Fig. 1. Schematic of the proposed SUD-aided and MUD-SIC-aided schemes. RNs in the SUD-aided scheme employ the SUD receivers, whereas RNs in the MUD-SIC-aided scheme employ the MUD-SIC receivers.

novel outage-optimal best effort-detection-based-ORS (BEDORS) scheme, which integrates the SIC algorithm into ORS. We demonstrate that the proposed BED-ORS is capable of achieving a better outage performance than the traditional ORS scheme originally designed for SUD receivers [8].

- To enhance the robustness of ORS considering the outdated CSI due to delayed feedback, we design an improved BEDORS (I-BED-ORS), which relies on the statistical information concerning the SN-DN and RN-DN channels to maximize the probability of avoiding an outage. We demonstrate that the I-BED-ORS is more robust against outdated CSI than BED-ORS.

The rest of this paper is organized as follows. The system model is introduced in Section II. In Section III, the outage analysis is detailed, followed by the proposed BED-ORS scheme, as well as our complexity analysis. In Section IV, our I-BED-ORS scheme relying on the outdated CSI is disclosed, whereas our simulation results are provided in Section V, followed by our conclusions in Section VI.

\section{System MODEL}

We consider a network composed of an SN, $K \mathrm{RNs}$, and a DN, as shown in Fig. 1, where $M$ interfering nodes (INs) may access the channel concurrently and introduce CCI. Each node is equipped with a single antenna and operates in a half-duplex mode. We assume a Rayleigh block fading-channel model, where the fading coefficient remains constant for the duration of a packet, and then it is faded independently from one packet to another both in time and space. The noise at the receivers is assumed to be independent zero-mean circularly symmetric complex Gaussian random variables.

At the RNs and the DN, the receiver has to detect the signals transmitted from the desired node, which may be sent by the $\mathrm{SN}$ in the broadcast phase and by the RNs selected for the retransmission phase. The SUD and MUD-SIC receivers are assumed to have perfect CSI for detection and decoding. In the MUD-SIC receiver, both the desired signal and the interfering signals are extracted from the composite received signal. In each iteration, the SIC decoder may remove the interference imposed by the successfully decoded signals from the current composite received signal. This process relies on re-encoding the decoded bit sequence and then remodulating it with the appropriate amplitude and phase adjustment relying on perfect CSI and subtracting it from the interference-infected composite signal. The signal that cannot be error-freely decoded is not subtracted from the composite received signal. It is assumed that error propagation due to erroneous decoding can be avoided with the aid of the cyclic redundancy check (CRC). First, the SN broadcasts its message to all RNs and to the DN. A CRC check is used to test whether the decoding succeeds or fails at the DN. If the decoding is successful, an acknowledgment (ACK) flag is broadcasted by the DN and the SN would initiate the next packet's transmission after receiving the ACK signal. Otherwise, the DN broadcasts the negative-acknowledgment (NACK) flag.

All RNs that overhear the NACK signal from the DN attempt to decode the signal buffered during the SN's broadcast phase. If an RN successfully decodes, it is capable of regenerating the original message and therefore joins the decoding set $D_{s}$. Furthermore, each node in $D_{s}$ sends an ACK signal to the DN to indicate successful decoding and providing the DN with the opportunity to evaluate the CSI [3], based on which the DN implements ORS and selects a retransmission node. Then, the DN notifies the selected node with the aid of a feedback channel, where the node selected re-encodes the message and transmits it. Throughout this paper, it is assumed that the feedback channel and the ACK/NACK flags are error free.

\section{ORS DESIGN}

1) Outage Analysis: An outage is defined as the scenario where the received signal-to-interference-noise power ratio (SINR) experienced at the DN is lower than the predefined threshold $\gamma_{\text {th }}$ that has to be exceeded for successful decoding. When no combining techniques are invoked at the receiver and the erroneous packets are discarded, ${ }^{4}$ the outage probability $P_{l}$ is equal to the probability that both the direct transmission and the retransmission are in outage. Hence, we have $P_{l}=P_{e}^{1} P_{e}^{2}$, where $P_{e}^{1}$ denotes the packet error ratio (PER) of the direct transmission, whereas $P_{e}^{2}$ denotes the PER of the retransmission. As the classic P2P transmission is a basic building block of CARQ, we first present the P2P PER analysis and then we deduce the outage probability of CARQ based on the P2P PER analysis.

First, a MUD-SIC receiver is adopted at the DN in both the SUDaided and MUD-SIC-aided systems. The per-user PER performance of a multiuser uplink system using a MUD-SIC receiver at the DN was characterized in [16] in the absence of relaying, where the uplink rate of each user was assumed to be identical. More specifically, the PER of each user is presented in [16] using the optimal decoding order, where the signal having the highest SNR is decoded, re-encoded, remodulated, and subtracted in each decoding iteration. A tight upper bound of the per-user PER is deduced in (28) of [16]. In our system, we focus our attention only on the PER performance of the desired user. We set the index of the SN to 0 , and $P_{e}^{1}$ is given in (1), where $\bar{\gamma}_{0}$ represents the average SNR of the SN at the DN, whereas $\bar{\gamma}_{1}, \ldots \bar{\gamma}_{M}$ represents the average interference-to-noise power ratio (INR) of the $M$ interferers at the $\mathrm{DN}$, respectively

$$
\begin{aligned}
P_{e}^{1} & =P_{e}^{\mathrm{MUD}-\mathrm{SIC}}\left(\bar{\gamma}_{0}, \bar{\gamma}_{1}, \ldots \bar{\gamma}_{M}\right) \\
& \leq \frac{1}{(M+1) !} \sum_{\pi}\left[\sum_{i=0}^{\pi^{-1(0)}}\left(1-\sum_{v=j}^{M} A_{v} e^{\frac{-\gamma_{\mathrm{th}}}{\lambda_{v}}}\right)\right] \prod_{i=0}^{M} \frac{1}{\phi_{i} \bar{\gamma}_{i}}
\end{aligned}
$$

\footnotetext{
${ }^{4}$ Please note that this is for brevity of outage analysis and to highlight the gist of BED-ORS proposed. If maximum-ratio combining is invoked, the expression in (2) and the correspondent BED-ORS should be modified to exploit the information in erroneous receptions. This is left for future works because of the limited space.
} 
The definitions of the parameters used are defined as follows: $\phi_{i}=\left(\sum_{u=1}^{i} \bar{\gamma}_{u}^{-1} / i\right), \quad \bar{\lambda}_{v}=\left(1-(v-j) \gamma_{\mathrm{th}}\right) /\left(v \phi_{v}\right), \quad$ and $A_{v}=$ $\prod_{u=j, u \neq v}^{n}\left(\bar{\lambda}_{v} /\left(\bar{\lambda}_{v}-\bar{\lambda}_{u}\right)\right)$.

Explicitly, $\pi$ represents the possible decoding order of the SIC, whereas $\pi(i)=0$ indicates that the desired signal is decoded during the $i$ th decoding iteration. ${ }^{5}$ Therefore, in the presence of $M$ interferers, there are $(M+1)$ ! possible decoding orders. Owning to lack of space, in this compact letter, we are unable to elaborate further on the detailed deduction of (1), which may be found in [16].

On the other hand, the PER of the retransmission phase $P_{e}^{2}$ may be expressed as

$$
P_{e}^{2}=\sum_{D_{s}} \operatorname{Pr}\left\{D_{s}\right\} P_{e}\left(D_{s}, \bar{\gamma}_{1}, \ldots, \bar{\gamma}_{M}\right)
$$

where $\operatorname{Pr}\left\{D_{s}\right\}$ is the probability that $D_{s}$ is the decoding set in the broadcast phase, which may be formulated as $\operatorname{Pr}\left\{D_{s}\right\}=\prod_{i \in D_{s}}(1-$ $\left.P_{e, s i}\right) \prod_{j \notin D s} P_{e, s j}$, where $i$ and $P_{e, s i}$ denote the $i$ th node in the decoding set and the error probability of its reception during the SN's broadcast phase, respectively. In this paper, the SUD-aided system treats the interference as noise at the RNs, and the corresponding $P_{e, s i}$ is given by [17]. The $P_{e, s i}$ of the MUD-SIC-aided system is in the form of (1), whereas the SNR and the INRs should be replaced by the values at the $i$ th RN. Therefore, the $\operatorname{Pr}\left\{D_{s}\right\}$ in (2) depends only on SN-RN channel quality and the receiver adopted at the RNs, namely, SUD or MUD-SIC receivers. Therefore, $\operatorname{Pr}\left\{D_{s}\right\}$ does not depend on the ORS design. On the contrary, the expression of $P_{e}\left(D_{s}, \bar{\gamma}_{1}, \ldots, \bar{\gamma}_{M}\right)$ in (2) depends on the ORS design, where $D_{s}$ is the decoding set and $\bar{\gamma}_{1}, \ldots, \bar{\gamma}_{M}$ represent the average INR of the $M$ interferers at the DN.

An outage-optimal ORS scheme achieves an outage probability $P_{e}^{\text {opt }}\left(D_{s}, \bar{\gamma}_{1}, \ldots, \bar{\gamma}_{M}\right)$, which is equal to the probability that transmissions from all the nodes in the decoding set $D_{s}$ to the DN failed [3]. However, $P_{e}^{\mathrm{opt}}\left(D_{s}, \bar{\gamma}_{1}, \ldots, \bar{\gamma}_{M}\right)$ cannot be simply decomposed into the form of $\prod_{i \in D_{s}} P_{e, i d}\left(\bar{\gamma}_{\mathrm{id}}, \bar{\gamma}_{1}, \ldots, \bar{\gamma}_{M}\right)$ as adopted in [3], where $P_{e, i d}\left(\bar{\gamma}_{\mathrm{id}}, \bar{\gamma}_{1}, \ldots, \bar{\gamma}_{M}\right)$ denotes the error probability at the DN when the $i$ th node of the decoding set is selected for transmission. This is because, although the ORS may rely on mutually independent SNRs as determined by $\bar{\gamma}_{\text {id }}$ and the independent fading of the current packet, the INRs represented by $\gamma_{1}, \ldots, \gamma_{M}$ are fixed for the fading realizations of the current packet, regardless of which node is selected from the decoding set. Therefore, this results in correlated error probabilities $P_{e, i d}\left(\bar{\gamma}_{\text {id }}, \bar{\gamma}_{1}, \ldots, \bar{\gamma}_{M}\right)$ for each node in the decoding set, when the MUD-SIC receivers are adopted at the DN. Therefore, it remains an open challenge to deduce the closed-form expression of $P_{e}^{\text {opt }}\left(D_{s}, \bar{\gamma}_{1}, \ldots, \bar{\gamma}_{M}\right)$, which is left for our future investigation. Instead, the focus of this paper is to conceive a beneficial ORS scheme, which achieves the optimal outage probability, when the MUD-SIC receiver is adopted at the $\mathrm{DN}$.

2) BED-ORS Design: The design criterion of ORS is to achieve the optimal $P_{e}^{\mathrm{opt}}\left(D_{s}, \gamma_{1}, \ldots, \gamma_{M}\right)$, which denotes the probability that transmission from all the nodes in $D_{s}$ to the DN failed. Therefore, an outage-optimal ORS should satisfy the following condition: If the specific retransmission by the node selected from set $D_{s}$ cannot be successfully decoded at the DN, then a retransmission by any other node picked from set $D_{s}$ would not be decoded successfully either. ${ }^{6}$

In the presence of multiple interferers at DN and when the SUD decoder is employed at the $\mathrm{DN}$, the optimal relay selection scheme is

\footnotetext{
${ }^{5}$ Note that the first iteration is indexed by 0 .

${ }^{6}$ It should be noted that, although we use the terminology of relay selection, the SN itself may be selected for retransmission as well, if no RNs decoded the message successfully during the broadcast phase or if the SN's retransmission may facilitate a better decoding result at DN compared with the RNs.
}

the same as that of the interference-free scenario [3] because the ORS scheme simply appoints the node having the highest instantaneous SNR at the DN [8]. This results in the highest instantaneous received SINR $Y_{\text {raw }}=\left(\gamma_{\mathrm{id}} /\left(\sum_{j=1}^{M} \gamma_{j}\right)\right)$, which is denoted as raw SINR at the $\mathrm{DN}$ and therefore achieves the lowest outage probability. However, if the more powerful MUD-SIC receiver is employed at the DN, the outage probability performance of relay selection is no longer determined by the raw SINR. This is due to the fact that some interfering signals may be decoded and removed from the composite signal, and hence, the effective SINR of the desired signal may be iteratively enhanced and expressed as $Y_{\text {eff }}=\left(\gamma_{\mathrm{id}} /\left(\sum_{j=k+1}^{M} \gamma_{j}\right)\right)$, where $k$ indicates that the desired signal is decoded in the $k$ th iteration, given that the previous $(k-1)$ interfering signals are successfully decoded. The SIC scheme relies on the power-based ranking of all-received signal components, and it is at its best when the received power values are clearly distinguishable. Therefore, appointing the node having the highest SNR at the DN for retransmission may be detrimental because the MUD-SIC receiver of the DN cannot reliably detect and decode the desired signal or the interference. Instead, we propose the BEDORS scheme based on the best effort detection criterion, which is implemented in Algorithm 1.

The philosophy of the BED-ORS is as follows. Retransmission by a node in decoding set $D_{s}$ may result in one of two decoding outcomes at the DN, i.e., success or failure. If the DN has the perfect knowledge of CSI for the links between each node in the decoding set and the $\mathrm{DN}$, as well as that of the links between the interferers and the DN, it may predict the decoding outcome of every node in the decoding set by implementing SIC testing, as described in Algorithm 1. Hence, the node that facilitates successful decoding at the DN may be selected.

\section{Algorithm 1: Best Effort Detection Based ORS (BED-ORS)}

1) INR ordering: Following the broadcast phase, we assume that $(L-1)$ RNs successfully decode the SN's message. Hence, there are $L$ candidate nodes in $D_{s}$, including the $\mathrm{SN}$. We denote the decoding set $D_{s}$ as $\left\{c_{1}, c_{2}, \ldots, c_{L}\right\}$. The $\mathrm{DN}$ ranks the received INR of the $M$ interfering signals $\left\{\gamma_{1}, \gamma_{2}\right.$, $\left.\ldots, \gamma_{M}\right\}$ in descending order, and the reordered INR queue is denoted as $\left\{\gamma_{(1)}, \gamma_{(2)}, \ldots, \gamma_{(M)}\right\}$, where $\gamma_{(1)} \geq \gamma_{(2)} \geq \ldots$ $\geq \gamma_{(M)}, \forall i \in[1, M]$. Then, for each node in $D_{s}$, the DN carries out SNR ordering and SIC testing, as described next.

2) SNR ordering: The DN selects a candidate node $c_{l}$ from $D_{s}$ and inserts its received SNR $\gamma_{c_{l}}$ in the INR queue, keeping the new queue, which is referred to as SIC queue $\gamma_{(1)} \geq$ $\gamma_{(2)} \geq \cdots \geq \gamma_{(M+1)}$ in a descending order. This is the optimal decoding order for equal-rate scenario, which is proved in [16].

3) SIC testing: First, for each position $i$ in the INR queue, an SNR upper threshold $\gamma_{i}^{u}$ and an SNR lower threshold $\gamma_{i}^{l}$ are calculated as $\gamma_{i}^{u}=\left(\gamma_{(i-1)} / \gamma_{\mathrm{th}}\right)-\sum_{j=i}^{M} \gamma_{(j)}-1$ and $\gamma_{i}^{l}=$ $\gamma_{\text {th }}\left(\sum_{j=i}^{M} \gamma_{(j)}+1\right)$ for the SIC testing step, where $\gamma_{\text {th }}$ is the predefined threshold to be exceeded for successful decoding. The bounds are derived as follows: If the SNR of a candidate node is inserted at position $i$, to facilitate successful decoding, a necessary condition is that we have $\left(\gamma_{(i-1)} /\left(\gamma_{c_{l}}+\right.\right.$ $\left.\left.\sum_{j=i}^{M} \gamma_{(j)}+1\right)\right) \geq \gamma_{\mathrm{th}}$ and $\left(\gamma_{c_{l}} /\left(\sum_{j=i}^{M} \gamma_{(j)}+1\right)\right) \geq \gamma_{\mathrm{th}}$, where the first inequality ensures that the SINR of the signal at position $(i-1)$ exceeds $\gamma_{\mathrm{th}}$; therefore, it is decoded successfully. By contrast, the second inequality ensures that the SINR of the candidate's signal exceeds $\gamma_{\mathrm{th}}$. Please note that the condition is not a sufficient condition because it also relies on the decoding outcomes of the preceding signals in the SIC queue. 


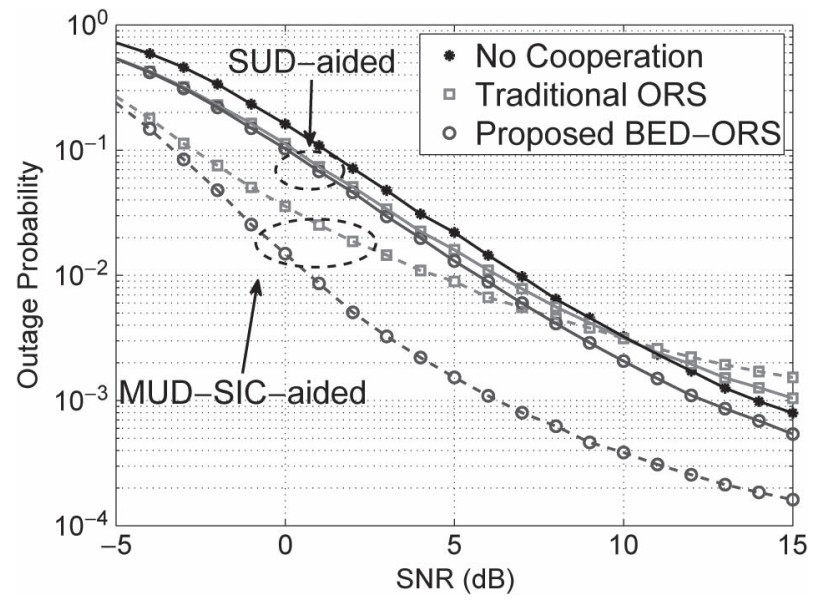

Fig. 2. Outage probability of different schemes versus the SN-DN link SNR for transmissions over block Rayleigh fading channels for $S I R=0 \mathrm{~dB}$, $\boldsymbol{R}_{T}=0.5 \mathrm{bit} / \mathrm{s} / \mathbf{H z}$, two RNs and four INs. The traditional ORS scheme is based on the highest SNR criteria, whereas the proposed BED-ORS scheme is implemented by Algorithm 1 assuming perfect CSI.

Denoting that after SNR ordering, $\gamma_{c_{l}}$ is inserted at position $k$. A failure is declared when the SINR of the signal in the $i$ th $(i<k)$ decoding iteration does not exceed $\gamma_{\mathrm{th}}$; therefore, none of the signals following in the queue (including the candidate signal) can be decoded because their SINR is even lower. Otherwise, if each $i$ th signal $(i \leq k)$ in the SIC queue is successfully decoded and $\gamma_{k}^{u} \geq \gamma_{c_{l}} \geq \gamma_{k}^{l}$ is satisfied, a successfully decoding outcome may be declared.

4) Node selection: If the decoding outcome predicted for $c_{l}$ is a success, $c_{l}$ is selected as the transmitting node and the node's index is sent by the DN through a feedback channel to the wouldbe-transmit node. Otherwise, the DN selects another candidate node that has not been tested yet and repeats steps 2 and 3. If decoding outcome predicted for all nodes in $D_{s}$ is a failure, an outage event is declared.

The proposed BED-ORS tests all candidates in $D_{s}$ until the decoding outcome of a candidate is successful and hence selected, or no candidates output a successful decoding outcome. Therefore, it is outage optimal by ensuring that if the node selected cannot facilitate successful decoding, any other nodes in $D_{s}$ would not be decoded successfully either. It should be noted that the proposed BEDORS does not involve the MUD-SIC decoding process. The DN may utilize MUD-SIC to decode the bits or symbols at the physical layer to recover the information transmitted. However, the BED-ORS is a MAC layer algorithm, which imitates the MUD-SIC process and relies only on processing of the SNRs and INRs. From the physical layer's perspective, the channel state information of the channels between all RNs and the DN and those of the channels between all INs and the DN are required by the MUD-SIC detecting stage. However, the physical layer SNRs and INRs have to be fed into the MAC layer and then exploited at the ORS stage, as it will be shown later in the simulation results in Fig. 2.

To support SIC decoding at the physical layer, the MAC layer of the ORS scheme originally proposed in [3] should be redesigned. The DN has the knowledge of the interference power; hence, it may serve as the central controller, which requires the CSI of the channels spanning from all candidate RNs to the DN. Therefore, we may summarize the MAC layer design conceived for our BED-ORS as follows. After the $\mathrm{SN}$ and DN communicate by exchanging their request-to-send and clear-to-send messages, then a NACK message is sent by the DN if the $\mathrm{DN}$ does not decode the data successfully. If an $\mathrm{RN}$ decodes the data, it sends a relay-request-to-send (RRTS) message and the DN hence becomes capable of estimating the channel spanning from all candidate RNs to the DN. Then, the relay selection is carried out by using Algorithm 1. Finally, the DN sends a relay-clear-to-send message, which includes the selected RN's identity.

Since in the MAC layer protocol proposed in [3], no interference is considered, the RNs contend to access the channel by setting up distributed timers at the RNs, where the RN having the highest SNR at the DN may be selected. Therefore, the contention period is independent of the number of RNs equipped with back-off timers. However, when there is CCI at the DN and the MUD-SIC is adopted, a practical issue arises from the fact that the CSI of the channels spanning from all candidate RNs to the DN has to be known at the DN. Therefore, each RN has to send a RRTS message to the DN for CSI estimation; hence, the total period required for the RRTS messaging is proportional to the number of candidate RNs. Therefore, the number of participating RNs should be appropriately controlled to avoid exceedingly high overall delays during the relay selection phase.

3) Complexity Analysis: Compared with the traditional ORS scheme based on the highest received SNR/SINR [3], [8], the proposed BED-ORS imposes higher complexity at the DN. Assuming $K \mathrm{RNs}$ and $M$ INs, the worst case in terms of the ORS implementation complexity is that the decoding set includes $(K+1)$ candidates in $D_{s}$, where all RNs decode successfully after the broadcast phase. Considering the merge sorting algorithm [18], the INR ordering of Algorithm 1 requires approximately $M \log _{2} M-M+1$ comparison operations, whereas the associated threshold calculation requires $2(M+1)$ division operations and $(M-1)$ addition operations. The worst case complexity of the SNR-based ordering requires $(K+1) \log _{2} M$ comparison operations to insert a candidate's SNR into the INR queue $(K+1)$ times. $^{7}$ SIC testing requires two comparison operations. Therefore, the overall number of computations for Algorithm 1's worst case scenario are $(K+M+1) \cdot \log _{2} M-M+2(K+1)+1$ comparison operations, $2(M-1)$ additions, and $2(M+1)$ divisions. In comparison, the traditional ORS scheme [3], [8] requires at worst $K$ comparisons to select the node from $D_{s}$ having the highest SNR. For example, for $K=M=4$, the numbers of comparison, addition, and division operations required by Algorithm 1 in the worst case are approximately 25,6 , and 10 , respectively, whereas the traditional ORS scheme requires at worst four comparison operations.

It should be noted that, although the BED-ORS introduces higher implementation complexity, it is negligible compared with decoding complexity imposed by the decoding process at the physical layer. For example, a state-of-art low-complexity design of a turbo decoder [19] requires multiple add-compare-select (ACS) operations per bit per iteration. Assuming a frame length of 1000 bits, a turbo decoder using four ACS operations, and four iterations, then it requires 8000 ACS operations for decoding a frame. In contrast, as BED-ORS is based on a frame-by-frame mechanism, it requires 25 comparison, 6 addition, and 10 division operations for a frame, assuming a scenario of four RNs and four INs. Therefore, the BED-ORS involves much lower complexity than the decoding process. We considered the classic cellular uplink scenario. If fixed RNs are installed by the operators, the RNs may indeed have sufficient processing capability to adopt MUD-SIC receivers. On the other hand, if user cooperation is adopted, where the RNs are mobile users, our SUD receivers may be adopted at the RN. Although the proposed ORS scheme imposed increased implementation complexity, it may exhibit significant outage probability improvements, as evidenced in Section V, where the implementation issues are discussed in more detail.

${ }^{7}$ All $K$ RNs successfully decode the SN's signal and join $D_{s}$; therefore, the size of $D_{s}$ is $(K+1)$. 


\section{OPTIMIZATION FOR OUTDATED CSI}

In the previous discussions, perfect CSI was assumed available at the RNs and the DN in the BED-ORS scheme. However, if the channel's coherence time is shorter than the time required for processing the relay selection plus the delay of feeding back the selected node index by the DN, the CSI used for ORS may become outdated and hence may be different from the actual CSI. As a result, the accuracy of the predictions may degrade. In this case, if the predicted decoding outcomes of multiple nodes in the decoding sets are a success, it is optimal to select the outcome where the probability that the actual decoding outcome being a success is maximized. Therefore, it should be emphasized that with outdated CSI, the ORS scheme is not guaranteed to be outage optimal. Alternatively, we may design an ORS scheme that maximizes the probability of avoiding outage or minimizes the probability of outage [15]. Therefore, we define the probability of avoiding an outage of candidate $c_{l}$ as the probability that the actual decoding outcome is a success, conditioned on the prediction being a success, denoted as $P_{f}^{c_{l}}$. The candidate $c_{\star}$ having the highest probability of avoiding an outage is selected as follows: $c_{\star}=\arg \max _{c_{l} \in D_{s}}\left\{P_{f}^{c_{l}}\right\}$.

To acquire $P_{f}^{c_{l}}$ for each $c_{l} \in D_{s}$, we have to evaluate the impact of outdated CSI. Using a similar approach as in [12], the correlation coefficient between the actual channel coefficient $h$ and the outdated channel coefficient $\hat{h}$ is defined as $\rho$. Therefore, we have [12]: $h=$ $\rho \hat{h}+\sqrt{1-\rho^{2}} v$, where $v \sim C N(0,1)$ and $\rho$ is assumed to be a constant in this paper. The actual SNR $\gamma$ conditioned on its estimate $\hat{\gamma}$ follows a noncentral chi square distribution with two degrees of freedom, and the probability density function of the SNR is expressed as [12]:

$$
f_{\gamma \mid \hat{\gamma}}(\gamma \mid \hat{\gamma})=\frac{1}{\bar{\gamma}\left(1-\rho^{2}\right)} e^{-\frac{\gamma+\rho^{2} \hat{\gamma}}{\bar{\gamma}\left(1-\rho^{2}\right)}} I_{0}\left(\frac{2 \rho \sqrt{\gamma \hat{\gamma}}}{\bar{\gamma}\left(1-\rho^{2}\right)}\right)
$$

where $\bar{\gamma}$ denotes the average $\mathrm{SNR}$, and $I_{0}(\cdot)$ is the zero-order modified Bessel function of the first kind. In our analysis, we assume that the CSIs of the candidates in the decoding set are outdated, whereas the CSIs of the interferers are perfect. Therefore, $\gamma_{i}^{l}$ and $\gamma_{i}^{u}$ defined in Step 1 of Algorithm 1 are not outdated. Then, the candidate $c_{l}$ 's probability of avoiding an outage $P_{f}^{c_{l}}$ may be expressed as [20]

$$
\begin{aligned}
P_{f}^{c_{l}} & =\operatorname{Pr}\left\{\gamma_{k}^{l} \leq \gamma_{c_{l}} \mid \hat{\gamma}_{c_{l}}\right\} \operatorname{Pr}\left\{\gamma_{c_{l}} \leq \gamma_{k}^{u} \mid \hat{\gamma}_{c_{l}}\right\} \\
\operatorname{Pr}\left\{\gamma_{c_{l}} \leq \gamma_{k}^{u} \mid \hat{\gamma}_{c_{l}}\right\} & =\int_{0}^{\gamma_{k}^{u}} f_{\gamma_{c_{l}} \mid \hat{\gamma}_{c_{l}}}\left(\gamma_{c_{l}} \mid \hat{\gamma}_{c_{l}}\right) d \gamma_{c_{l}} \\
& =1-Q_{1}\left(\lambda, b_{u}\right) \\
\operatorname{Pr}\left\{\gamma_{k}^{l} \leq \gamma_{c_{l}} \mid \hat{\gamma}_{c_{l}}\right\} & =1-\int_{0}^{\gamma_{k}^{l}} f_{\gamma_{c_{l}} \mid \hat{\gamma}_{c_{l}}}\left(\gamma_{c_{l}} \mid \hat{\gamma}_{c_{l}}\right) d \gamma_{c_{l}} \\
& =Q_{1}\left(\lambda, b_{l}\right)
\end{aligned}
$$

where $Q_{1}(\lambda, b)$ is the Marcum Q-function of order 1, whereas $\lambda, b_{u}$ and $b_{l}$ are defined as [20]: $\lambda=\rho \sqrt{\left(2 \hat{\gamma}_{c_{l}} /\left(\bar{\gamma}_{c_{l}}\left(1-\rho^{2}\right)\right)\right)}$, $b_{u}=\left(2 \gamma_{k}^{u} /\left(\bar{\gamma}_{c_{l}}\left(1-\rho^{2}\right)\right)\right)^{2}$, and $b_{l}=\left(2 \gamma_{k}^{l} /\left(\bar{\gamma}_{c_{l}}\left(1-\rho^{2}\right)\right)\right)^{2}$. Hence, to achieve the lowest outage probability, Algorithm 1 may be revised as follows:

\section{Algorithm 2: I-BED-ORS using outdated-CSI}

1) INR ordering: The same procedure of INR ordering is implemented as in Algorithm 1.

2) SNR ordering: The DN selects a candidate node $c_{l}$ and the same procedure of SNR ordering is implemented as in Algorithm 2.
3) SIC testing: The same procedure of SIC testing is implemented as in Algorithm 2. Additionally, the probability of avoiding an outage $P_{f}^{c_{l}}$ is calculated using (4)-(6).

4) Node selection: For every candidate node $c_{l}$ in $D_{s}$, the DN estimates the decoding outcomes and selects the node $c_{l^{\star}}$ of highest probability of avoiding an outage obeying $c_{l^{\star}}=$ $\max _{c_{l} \in D_{s}} P_{f}^{c_{l}}$. Then, this node's index is sent by the DN to the relevant $\mathrm{RN}$ via a feedback channel.

As shown in (4)-(6), the calculation of probability of avoiding an outage $P_{f}^{c_{l}}$ involves integration and may be computational expensive. As $\rho$ and $\bar{\gamma}_{c_{l}}$ are statistical parameters, which are assumed to be known so that $\lambda$ is known at the DN, a lookup table connecting the values of $Q_{1}(\lambda, b)$ and $b$ may facilitate acceptable implementation complexity. ${ }^{8}$

\section{Simulation Results and Discussions}

Here, we evaluate the performance of the two proposed systems in terms of the associated packet loss ratio (outage probability). In all our simulations, we consider a topology comprising $K$ RNs, all placed halfway along the line between the SN and DN, and we set the channel's path loss exponent to 3 . Additionally, we assume that all nodes in the network use an identical transmit power value. The time-average SNR of the SN-DN channel is denoted as $\bar{\gamma}_{s, d}$. Therefore, the SNR of the channel between any two nodes is $\bar{\gamma}_{i, j}=\left(d_{i, j} / d_{s, d}\right)^{-\alpha} \bar{\gamma}_{s, d}$, where $d_{i, j}$ denotes the distance between node $i$ and node $j$ and $\alpha$ is the channel's path loss exponent. The channels are assumed to be subjected to independent Rayleigh block fading. Throughout the simulations, the transmission rate of each $\mathrm{SN}$ is $R_{T}=0.5 \mathrm{bit} / \mathrm{s} / \mathrm{Hz}$.

4) Effects of ORS Criteria: We compare the outage probability performance of the traditional ORS scheme based on the highest received SNR/SINR criterion to that of the proposed BED-ORS scheme based on the best effort detection criterion described in Algorithm 1. It is also assumed that the average signal-to-interference power ratio (SIR) for each IN is $0 \mathrm{~dB} .{ }^{9}$ The number of RNs and INs is two and four, respectively. Fig. 2 shows the outage probability of the proposed MUD-SIC-aided CARQ system and of the SUD-aided CARQ system. The traditional ORS in Fig. 2 indicates a simple combination of the MUD-SIC with ORS relying on [3], which does not exploit the INRs during the relay selection process. When the traditional ORS scheme based on the highest received $S N R$ criterion is used, it is observed that in Fig. 2, a small improvement in outage probability performance is achieved by the MUD-SIC-aided CARQ system and SUD-aided CARQ system over the noncooperative system when the SN-DN SNR is within the range from $-5 \mathrm{~dB}$ to $-10 \mathrm{~dB}$. However, this trend no longer holds for higher SN-DN SNRs, where the employment of cooperation seems to be detrimental in terms of the outage probability performance, as shown in Fig. 2.

The reason for this behavior was provided in Section III. In this case, selecting an RN having a lower SNR at the DN may be preferred, where the strong interfering signals may be successfully decoded and subtracted, resulting in an improved SINR for the desired signal and facilitating a higher probability of successful decoding, which is the philosophy behind the BED-ORS scheme. Fig. 2 shows that both the MUD-SIC-aided and SUD-aided systems using BED-ORS scheme

\footnotetext{
${ }^{8}$ More practically, even if $\rho$ and $\bar{\gamma}_{c_{l}}$ are unknown at the DN, a heuristic method may be implemented by selecting $c_{l^{\star}}=\max _{c_{l} \in D_{s}} \min \left(\gamma_{k}^{u}-\right.$ $\left.\gamma_{c_{l}}, \gamma_{c_{l}}-\gamma_{k}^{l}\right)$.

${ }^{9}$ The average SIR is measured at the RNs in the broadcast phase, whereas at the DN, the SIR in the broadcast phase for current setting is approximately $-9 \mathrm{~dB}$. For simplicity, we use SIR at RNs in the broadcast phase to measure interference power.
} 


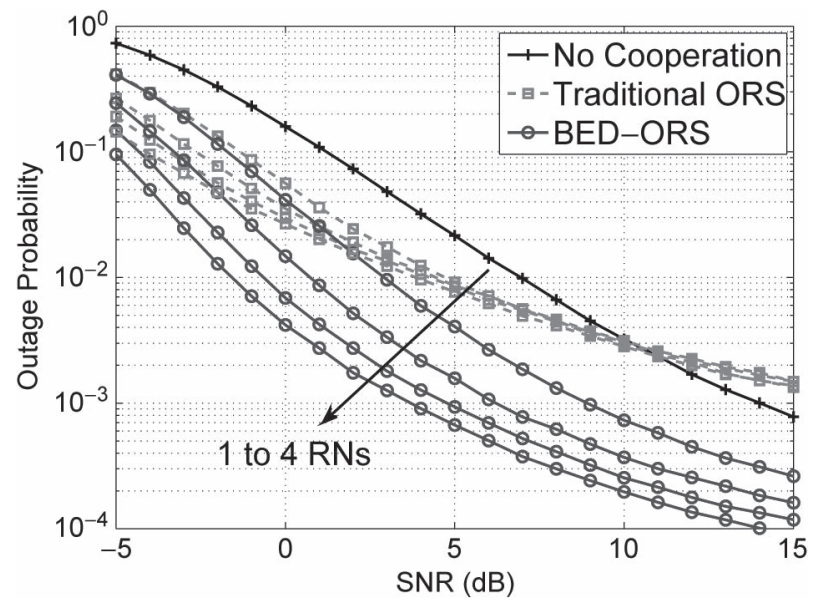

Fig. 3. Outage probability versus the SN-DN link SNR for transmissions over block Rayleigh fading channels with SIR $=0 \mathrm{~dB}, R_{T}=0.5 \mathrm{bit} / \mathrm{s} / \mathrm{Hz}$, four INs, and one to four RNs. The MUD-SIC-aided scheme is adopted. The traditional ORS scheme is based on the highest SNR criteria, whereas the proposed BEDORS scheme is implemented by Algorithm 1 assuming perfect CSI.

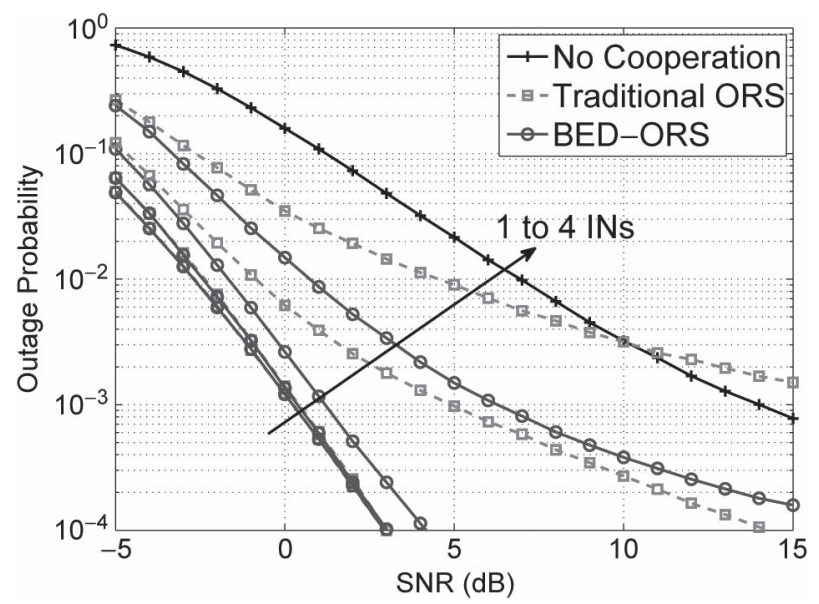

Fig. 4. Outage probability versus the SN-DN link SNR for transmissions over block Rayleigh fading channels with $\mathrm{SIR}=0 \mathrm{~dB}, R_{T}=0.5 \mathrm{bit} / \mathrm{s} / \mathrm{Hz}$, two RNs, and one to four INs. The MUD-SIC-aided scheme is adopted. The traditional ORS scheme is based on the highest SNR criteria, whereas the proposed BED-ORS scheme is implemented by Algorithm 1 assuming perfect CSI.

implemented in Algorithm 1 outperform the noncooperative system for all the SNRs considered. At $P_{l}=10^{-3}$, a substantial gain of about $7.5 \mathrm{~dB}$ is achieved by the MUD-SIC-aided system relying on two $\mathrm{RNs}$ over the noncooperative system, whereas only a $1.8-\mathrm{dB}$ gain is achieved by the SUD-aided system.

5) Effects of Number of Relays, Interferer, and Interference Power: Then, we evaluate the performance of our proposed systems for different numbers of RNs and INs. First, the number of INs is fixed to four, and the number of RNs is increased from one to four. It is observed in Fig. 3 that, as the number of RNs increases, the outage performance of the MUD-SIC-aided system adopting the proposed BED-ORS scheme was gradually improved as a benefit of increased spatial diversity, which outperforms the traditional ORS scheme.

Then, the number of RNs is fixed to two, and the number of INs increases from one to four. It is observed in Fig. 4 that the proposed BED-ORS scheme always achieves better outage performance than the traditional ORS for different number of INs. However, when the number of INs is one or two, the outage performance of traditional

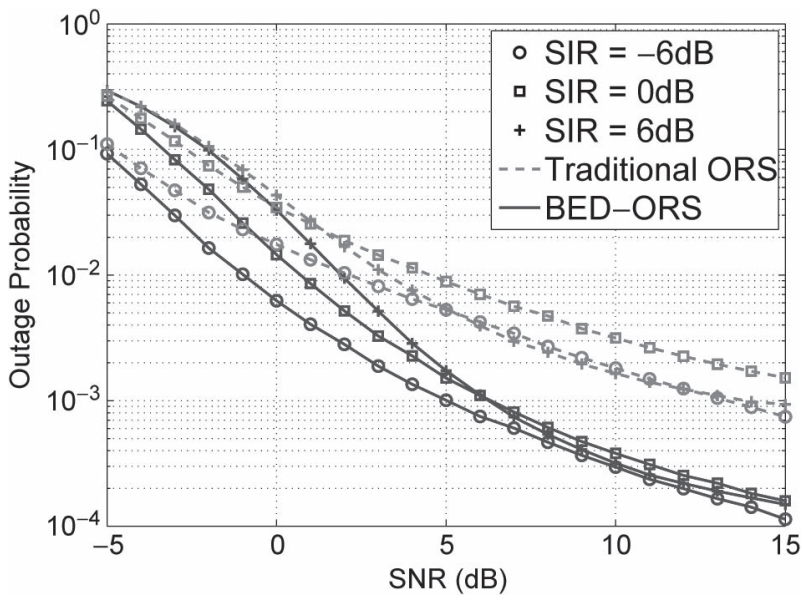

Fig. 5. Outage probability versus the SN-DN link SNR for transmissions over block Rayleigh fading channels with $S I R=-6,0$, and $6 \mathrm{~dB} ; R_{T}=$ $0.5 \mathrm{bit} / \mathrm{s} / \mathrm{Hz}$; two RNs; and four INs. The MUD-SIC-aided scheme is adopted. The traditional ORS scheme is based on the highest SNR criteria, whereas the proposed BED-ORS scheme is implemented by Algorithm 1 assuming perfect CSI.

ORS is very close to that of BED-ORS. The reason is that when the number of INs is relatively small and the resultant SINR is sufficiently high, the performance of a MUD-SIC receiver and a SUD receiver is identical.

The effects of interference power are evaluated in Fig. 5, where an $\mathrm{SN}$ assisted by two RNs and four INs is considered. We consider three signal-to-interference power levels, i.e., $S I R=0,6$, and $12 \mathrm{~dB}$, respectively. The dashed lines represent the performance of traditional ORS, whereas the solid lines represent the performance of the BEDORS under different SIR levels. It is observed that the BED-ORS scheme achieves better outage performance than traditional ORS. It is also observed that at low SNR region, a higher SIR may result in worse outage performance. The reason is that the probability of the interference being successfully decoded and removed is low due to the low INR. Assuming a constant SIR, as SNR increases, the INR correspondingly increases and therefore achieving higher probability of decoding and removing interference, which in turn results in improved outage performance.

On the other hand, Fig. 6 shows the importance of employing the more powerful MUD-SIC receiver at the DN in the presence of interference. If the DN adopts SUD receivers, the PLR performance improvement achieved by enhancing SN-DN link quality erodes at moderate SNRs and the system encounters the interference-limited region, as shown by the dotted lines. The proposed SUD- and MUDSIC-aided schemes, which are represented by the dashed and solid lines, respectively, are capable of achieving better outage performance, with the aid of MUD-SIC receivers at the DN. We may conclude that in the presence of interference, a DN adopting the MUD-SIC receiver is the guarantee of achieving a low outage probability. As the SUD receivers exhibit lower implementation decoding complexity than the MUD-SIC receivers, SUD-aided scheme may be preferred because of low-complexity RNs, provided that the outage performance degradation remains tolerable. Fig. 5 shows an example associated with $S I R=12 \mathrm{~dB}$, where the degradation of the SUD-aided scheme is approximately $1.2 \mathrm{~dB}$ at $P L R=10^{-3}$ compared with the MUDSIC-aided scheme.

Practically, the decoding complexity of the MUD-SIC increases at most linearly with the number of interferers. Therefore, we are capable of controlling the number of interferers actually decoded by the MUDSIC receivers. In other words, we may process a limited number 


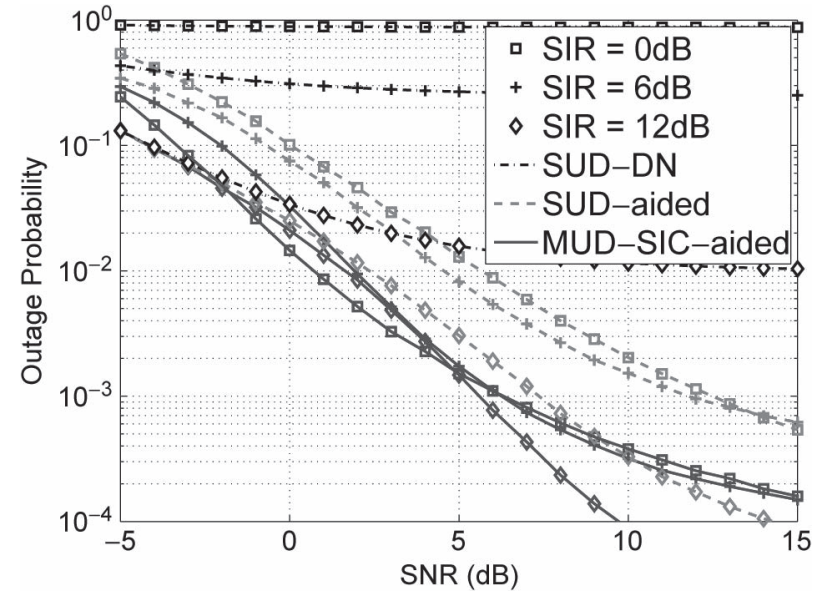

Fig. 6. Outage probability versus the SN-DN link SNR for transmissions over block Rayleigh fading channels with $S I R=0,6$, and $12 \mathrm{~dB} ; R_{T}=$ $0.5 \mathrm{bit} / \mathrm{s} / \mathrm{Hz}$; two RNs; and four INs. The MUD-SIC-aided and SUDsided schemes are compared. The BED-ORS scheme is implemented by Algorithm 1 assuming perfect CSI.

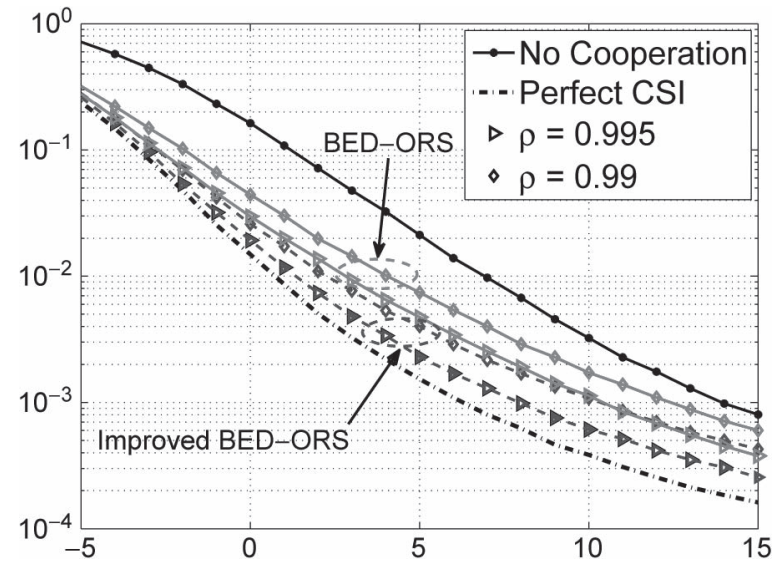

Fig. 7. Outage probability versus the SN-DN link SNR for transmissions over block Rayleigh fading channels with outdated CSI, where correlation coefficient $\rho=0.99,0.995$. SIR $=0 \mathrm{~dB}, R_{T}=0.5 \mathrm{bit} / \mathrm{s} / \mathrm{Hz}$, two RNs, and four INs. The BED-ORS is implemented by Algorithm 1, and I-BED-ORS is implemented by Algorithm 2. Only the performance of the MUD-SIC-aided CARQ is shown because it exhibits highest outage improvements toward the noncooperative counterparts.

of strong interferers using MUD-SIC while treating the remaining interferers as noise. In this way, we may remove the most detrimental components and strike a balance between the outage performance achieved and the complexity imposed.

6) Effects of Outdated CSI and Using I-BED-ORS: Fig. 7 shows the outage probability performance of the ORS proposed in Section IV. If the CSI is outdated and the DN employs the suboptimum Algorithm 1 based on perfect CSI, the outage probability performance may severely degrade, as shown in Fig. 7. By contrast, when the I-BED-ORS implemented in Algorithm 2 is used, the outage probability performance may be substantially improved, as shown in Fig. 7. However, as the correlation coefficient $\rho$ decreases and the reliability of the outdated CSI drops, the outage probability performance of the optimized ORS using Algorithm 2 degrades. It may be concluded that the accuracy of CSI used for ORS is critical for the achievable outage probability performance of cooperative systems adopting MUD-SIC receivers.

\section{CONCLUSION}

In this paper, we have considered two CARQ systems adopting MUD-SIC receivers at the DN, namely, the MUD-SIC-aided CARQ and SUD-aided CARQ schemes. The outage-optimal BED-ORS was proposed for both systems based on a best effort detection criterion. The proposed systems were shown to outperform both the noncooperative system and the system employing SUD receivers at the DN, whereas the MUD-SIC-aided system achieved significant outage probability performance improvements with the aid of the BEDORS. Additionally, an optimized BED-ORS scheme was designed, which was shown to have improved robustness against degraded CSI accuracy imposed by outdated CSI.

\section{REFERENCES}

[1] B. Zhao and M. Valenti, "Practical relay networks: A generalization of hybrid-ARQ," IEEE J. Sel. Commun., vol. 23, no. 1, pp. 7-18, Jan. 2005

[2] Y. Jing and H. Jafarkhani, "Single and multiple relay selection schemes and their achievable diversity orders," IEEE Trans. Wireless Commun., vol. 8, no. 3, pp. 1414-1423, Mar. 2009.

[3] A. Bletsas, H. Shin, and Z. Win, "Cooperative communications with outage-optimal opportunistic relaying," IEEE Trans. Wireless Commun., vol. 6, no. 9, pp. 3450-3460, Sep. 2007.

[4] I. Krikidis, T. Charalambous, and J. S. Thompson, "Buffer-aided relay selection for cooperative diversity systems without delay constraints," IEEE Trans. Wireless Commun., vol. 11, no. 5, pp. 1957-1967, May 2012.

[5] F. Etezadi, K. Zarifi, A. Ghrayeb, and S. Affes, "Decentralized relay selection schemes in uniformly distributed wireless sensor networks," IEEE Trans. Wireless Commun., vol. 11, no. 3, pp. 938-951, Mar. 2012.

[6] C.-H. Yu and O. Tirkkonen, "Opportunistic multiple relay selection with diverse mean channel gains," IEEE Trans. Wireless Commun., vol. 11, no. 3, pp. 885-891, Mar. 2012.

[7] I. Krikidis, J. Thompson, S. Mclaughlin, and N. Goertz, "Max-min relay selection for legacy amplify-and-forward systems with interference," IEEE Trans. Wireless Commun., vol. 8, no. 6, pp. 3016-3027, Jun. 2009.

[8] D. Lee and J. H. Lee, "Outage probability of decode-and-forward opportunistic relaying in a multicell environment," IEEE Trans. Veh. Technol., vol. 60, no. 4, pp. 1925-1930, May 2011.

[9] H. Yu, I.-H. Lee, and G. L. Stuber, "Outage probability of decodeand-forward cooperative relaying systems with co-channel interference,' IEEE Trans. Wireless Commun., vol. 11, no. 1, pp. 266-274, Jan. 2012.

[10] D. Tse and P. Viswanath, Fundamentals of Wireless Communication. Cambridge, U.K.: Cambridge Univ. Press, 2005.

[11] C. Gong, A. Tajer, and X. Wang, "Group decoding for multi-relay assisted interference channels," IEEE J. Sel. Areas Commun., vol. 30, no. 8, pp. 1489-1499, Sep. 2012.

[12] J. Vicario, A. Bel, J. Lopez-Salcedo, and G. Seco, "Opportunistic relay selection with outdated CSI: Outage probability and diversity analysis," IEEE Trans. Wireless Commun., vol. 8, no. 6, pp. 2872-2876, Jun. 2009.

[13] M. Chen, T. C.-K. Liu, and X. Dong, "Opportunistic multiple relay selection with outdated channel state information," IEEE Trans. Veh. Technol., vol. 61, no. 3, pp. 1333-1345, Mar. 2012.

[14] S. Kim, S. Park, and D. Hong, "Performance analysis of opportunistic relaying scheme with outdated channel information," IEEE Trans. Wireless Commun., vol. 12, no. 2, pp. 538-549, Feb. 2013.

[15] Y. Li, Q. Yin, W. Xu, and H.-M. Wang, "On the design of relay selection strategies in regenerative cooperative networks with outdated CSI," IEEE Trans. Wireless Commun., vol. 10, no. 9, pp. 3086-3097, Sep. 2011.

[16] V. Lau, "Per-user packet outage analysis in slow multiaccess fading channels with successive interference cancellation for equal rate applications," IEEE Trans. Wireless Commun., vol. 7, no. 5, pp. 1754-1763, May 2008.

[17] D. Lee and J. H. Lee, "Outage probability for dual-hop relaying systems with multiple interferers over Rayleigh fading channels," IEEE Trans. Veh. Technol., vol. 60, no. 1, pp. 333-338, Jan. 2011.

[18] T. H. Cormen, C. Stein, R. L. Rivest, and C. E. Leiserson, Introduction to Algorithms., 2nd ed. New York, NY, USA: McGraw-Hill, 2001.

[19] L. Li, R. G. Maunder, B. M. Al-Hashimi, and L. Hanzo, "A lowcomplexity turbo decoder architecture for energy-efficient wireless sensor networks," IEEE Trans. Very Large Scale Integr. (VLSI) Syst., vol. 21, no. 1, pp. 14-22, Jan. 2013.

[20] A. Nuttall, "Some integrals involving the Q function," IEEE Trans. Inf. Theory, vol. 21, no. 1, pp. 95-96, Jan. 1975. 